\title{
Quasi-Homogeneous Thermodynamics and Black Holes
}

\author{
F. Belgiorno* \\ Dipartimento di Fisica, Università degli Studi di Milano, Via Celoria 16, 20133 Milano, Italy
}

(July 6, 2021)

We propose a generalized thermodynamics in which quasi-homogeneity of the thermodynamic potentials plays a fundamental role. This thermodynamic formalism arises from a generalization of the approach presented in paper [1], and it is based on the requirement that quasi-homogeneity is a non-trivial symmetry for the Pfaffian form $\delta Q_{r e v}$. It is shown that quasi-homogeneous thermodynamics fits the thermodynamic features of at least some self-gravitating systems. We analyze how quasi-homogeneous thermodynamics is suggested by black hole thermodynamics. Then, some existing results involving self-gravitating systems are also shortly discussed in the light of this thermodynamic framework. The consequences of the lack of extensivity are also recalled. We show that generalized Gibbs-Duhem equations arise as a consequence of quasi-homogeneity of the thermodynamic potentials. An heuristic link between this generalized thermodynamic formalism and the thermodynamic limit is also discussed.

PACS: 05.70.-a, 04.70.Dy

*E-mail address: belgiorno@mi.infn.it 


\section{INTRODUCTION}

In paper [1] we have shown that, by choosing the extensive variables $\left(U, V, X^{1}, \ldots, X^{n}\right)$ as independent variables, and by postulating that the integrable Pfaffian form $\delta Q_{r e v}$ representing the infinitesimal heat exchanged reversibly is homogeneous of degree one and that the homogeneity symmetry is non-trivial [for the definition of non-trivial symmetry see the next section], it is possible to find immediately an integrating factor for $\delta Q_{r e v}$. In fact, if $Y=U \partial_{U}+V \partial_{V}+\sum_{i} X^{i} \partial_{X^{i}}$ is the Liouville vector field associated with the homogeneity symmetry, one has that an integrating factor is given by $f=\delta Q_{\text {rev }}(Y)=i_{Y} \delta Q_{\text {rev }} \not \equiv 0$ (see [1] and references therein). The entropy $S\left(U, V, X^{1}, \ldots, X^{n}\right)$, which represents the fundamental equation in the entropy representation, is then straightforwardly recovered. The framework presented in paper [1] can be generalized in the following sense. A generalized thermodynamics where $\delta Q_{r e v}$ is a quasi-homogeneous integrable Pfaffian form is proposed. Quasi-homogeneity is realized to be a property which characterizes the behavior of thermodynamic functions like e.g. the entropy in the black hole case, in some statistical mechanical models involving non-relativistic matter interacting via the Newtonian potential and also in the case of self-gravitating radiation. When gravity plays an important role the standard extensivity property of thermodynamics does not hold; nevertheless, one can still find a form of thermodynamics in which each thermodynamic variable follows a power scaling law where the power can be different from one or zero, i.e., the variables are allowed to be neither extensive nor intensive. Black hole thermodynamics is the most evident and special thermodynamics belonging to this framework.

The plan of the paper is the following. In sect. II we give some definitions and then in sect. III, we propose a theoretical framework for quasi-homogeneous thermodynamics, by generalizing the standard thermodynamics case [1]. In sects. IV,V and VI examples displaying a quasi-homogeneous thermodynamics are discussed. In sect. IV, the role of quasi-homogeneity in black hole thermodynamics is shown; in sect. V, we discuss a model of fermionic non-relativistic matter with Newtonian interaction which displays a quasi-homogeneous behavior. In sect. VI, the general relativistic case of thermal geons and self-gravitating radiation are discussed. Sect. VII concerns the discussion of the physical consequences for thermodynamics when the requirement of homogeneity is relaxed. In sect. VIII a summary of constructive assumptions is made. In sect. IX the generalized Gibbs-Duhem identities are discussed. In sect. X the energy representation and the Legendre transform are analyzed. In sect. XI some further suggestions from black hole thermodynamics are discussed. Sect. XII has an heuristic nature, a link between the thermodynamic limit and the formalism of quasi-homogeneous thermodynamics is proposed. In the Appendix further mathematical and physical aspects are explored.

\section{QUASI-HOMOGENEOUS PFAFFIAN FORMS}

Given a set of real coordinates $x \equiv\left(x^{1}, \ldots, x^{n}\right)$ and a set of weights $\boldsymbol{\alpha} \equiv\left(\alpha_{1}, \ldots, \alpha_{n}\right) \in \mathbb{R}^{n}$, a real-valued function $G\left(x^{1}, \ldots, x^{n}\right)$ is quasi-homogeneous of degree $r$ and type $\boldsymbol{\alpha}$ if, under the action of the one-parameter group of quasihomogeneous dilatations

$$
g^{\tau}:\left(x^{1}, \ldots, x^{n}\right) \rightarrow\left(\mathrm{e}^{\alpha_{1} \tau} x^{1}, \ldots, \mathrm{e}^{\alpha_{n} \tau} x^{n}\right),
$$

where $\tau \in \mathbb{R}$, one finds

$$
G\left(g^{\tau} x\right)=\mathrm{e}^{r \tau} G(x) .
$$

A differentiable function $G$ on a open connected domain

$$
\mathcal{D}=\left\{x \in \mathcal{D} \mid g^{\tau} x \in \mathcal{D} \forall x \in \mathcal{D}, \tau \in \mathbb{R}\right\} \subseteq \mathbb{R}^{n}
$$

is quasi-homogeneous of degree $r$ if and only if $[2,3]$

$$
D G=r G,
$$

where the Euler vector field

$$
D \equiv \alpha_{1} x^{1} \frac{\partial}{\partial x^{1}}+\ldots+\alpha_{n} x^{n} \frac{\partial}{\partial x^{n}}
$$

is the infinitesimal generator of the transformation. Notice that a quasi-homogeneous transformation is also called "similarity transformation" and "stretching transformation" [4]. The identity (4) is a generalization of the Euler identity for homogeneous functions. Quasi-homogeneity for functions and vector fields is defined in Ref. [2]. Notice that, 
according to the definition given above, one cannot avoid specifying, together with the degree of quasi-homogeneity, the type of the quasi-homogeneous object one is considering.

Homogeneous functions are a subset of quasi-homogeneous functions, in fact they have all the weights equal to one. We define strictly quasi-homogeneous functions the functions which satisfy the above definition with weights which cannot be all equal. A quasi-homogeneous function of $n$ variables is characterized by $n$ weights and its degree. If all the weights are undetermined and arbitrary, or if they are in part undetermined and in part equal to one, then all the weights can be put equal to one, and then the function is actually homogeneous. The viceversa is not true, because there are homogeneous functions which don't admit different weights (i.e., they are not strictly quasi-homogeneous). A trivial example is given by $g(x, y)=x^{2}+y^{2}$, which is homogeneous of degree two but it is not also strictly quasihomogeneous. Instead, the function $h(x, y)=x y$ is homogeneous of degree two and it is also quasi-homogeneous with undetermined weights. In the following, "quasi-homogeneous" is used as synonymous of strictly quasi-homogeneous, unless a more general discussion is required.

A Pfaffian form

$$
\omega=\sum_{i=1}^{n} \omega_{i}(x) d x^{i}
$$

is defined to be quasi-homogeneous of degree $r \in \mathbb{R}$ if, under the scaling

$$
x^{1}, \ldots, x^{n} \mapsto \lambda^{\alpha_{1}} x^{1}, \ldots, \lambda^{\alpha_{n}} x^{n}
$$

one finds

$$
\omega \mapsto \lambda^{r} \omega
$$

This happens if and only if the degree of quasi-homogeneity $\operatorname{deg}\left(\omega_{i}(x)\right)$ of $\omega_{i}(x)$ is such that $\operatorname{deg}\left(\omega_{i}(x)\right)=r-\alpha_{i} \quad \forall i=$ $1, \ldots, n$. Let us assume that a quasi-homogeneous $\omega$ is integrable, i.e. $\omega \wedge d \omega=0$. A quasi-homogeneous transformation is a symmetry for $\omega$ (see e.g. $[5,6])$, in the sense that

$$
\left(L_{D} \omega\right) \wedge \omega=0
$$

where $L_{D}$ is the Lie derivative associated with $D$ defined in (5). An integrating factor $f$ such that the form $\omega / f$ is closed can then be constructed by contracting the vector field $D$ with $\omega$ :

$$
f \equiv \omega(D)=i_{D}(\omega)
$$

and $\omega(D) \not \equiv 0$ is to be assumed. This means that the symmetry generated by $D$ is nontrivial, in the sense that it does not belong to the distribution of codimension one which is associated with the kernel of $\omega$. Then, the symmetry is not tangent to each leaf of the foliation associated with the integrable 1-form $\omega$, but it carries leaves onto other leaves. In this sense, a nontrivial symmetry is transversal with respect to the foliation.

One gets

$$
d\left(\frac{\omega}{f}\right)=0
$$

The integrating factor $f$ is a quasi-homogeneous function of degree $r$, because $D i_{D}(\omega)=i_{D} L_{D} \omega=r i_{D}(\omega)$ [due to the Cartan formula $L_{X} i_{Y}-i_{Y} L_{X}=i_{[X, Y]}$ and to $\left.i_{0}=0\right]$. Then $\omega / f$ is of degree zero and its integral can be found only by quadratures (cf. sect. III); one gets

$$
\hat{S}-\hat{S}_{0} \equiv \int_{\Gamma} \frac{\omega}{f}
$$

It can be shown that there exists a quasi-homogeneous function $F$ of degree one with respect to $D$ such that

$$
\begin{aligned}
\hat{S} & =\log (F) \\
D F & =F,
\end{aligned}
$$

thus it holds $D \hat{S}=1$ and $\hat{S}$ is not quasi-homogeneous. On this topic see Appendix A, where the case of a generic transversal symmetry is treated. The above construction is completely analogous to the construction developed for homogeneous integrable Pfaffian forms in Ref. [1]. 


\section{A GENERAL FRAMEWORK}

Quasi-homogeneous thermodynamics [recall that we mean strictly quasi-homogeneous] overcomes the standard distinction between extensive and intensive variables. We propose the following generalization for the integrable Pfaffian form $\delta Q_{\text {rev }}$ which represents the infinitesimal heat exchanged by the system:

$$
\delta Q_{\text {rev }}=d U^{*}-\sum_{i=1}^{n} \xi_{i}^{*} d X^{i *}
$$

where the asterisk indicates that both the independent variables $U^{*}, X^{1 *}, \ldots, X^{n *}$ and the dependent ones $\xi_{1}^{*}, \ldots, \xi_{n}^{*}$ in (15) are generalizations of the usual ones in the sense that they are not simply intensive and/or extensive but quasi-homogeneous, in such a way that the Pfaffian form $\delta Q_{r e v}$ is quasi-homogeneous of degree $r$. Moreover, for the sake of definiteness and in order to fit some requirements for the validity of Frobenius' theorem, $\delta Q_{r e v}$ is assumed to be of class at least $C^{1}$ in the thermodynamic domain (except, maybe, on the boundary as e.g. the surface $T=0$ ).

The thermodynamic domain $\mathcal{D}$ is assumed to be a simply connected set which satisfies (3). A further requirement for $\mathcal{D}$ has to be introduced if the entropy $S^{*}$ is required to be superadditive: $\mathcal{D}$ has to be closed with respect to the sum (see sect. VII for further details). Cf. also subsect. III B 1. [In standard homogeneous thermodynamics, the thermodynamic domain can be assumed to be a convex cone [1], which is a cone with the property to be closed under addition ( [7], pp. 13-14). One can also assume that the domain $\mathcal{D}$ is still a convex cone with the further requirement that it has to be invariant under quasi-homogeneous transformations. This can be obtained by considering a set $\mathcal{C} \subset \mathbb{R}^{n+1}$ which is invariant under quasi-homogeneous transformations and then the set $\mathcal{K}$ of all the positive linear combinations of elements of $\mathcal{C}$. The set $\mathcal{K}$ is the smallest convex cone containing $\mathcal{C}$ ( [7], p. 14). This cone $\mathcal{K}$ is trivially still invariant under quasi-homogeneous transformations.]

In the following, it is useful to refer to the variables $U^{*}, X^{1 *}, \ldots, X^{n *}$ as would-be extensive variables, and to the variables $\xi_{1}^{*}, \ldots, \xi_{n}^{*}$ (and also $T^{*}$ ) as would-be intensive variables. The variables $U^{*}, X^{1 *}, \ldots, X^{n *}$ could also be chosen to be such that they are additive, i.e., if one considers a system which is composed by two non-interacting subsystems, then $X^{*}=X_{1}^{*}+X_{2}^{*}$. See also Ref. [8].

We assume that the $X^{i *}$ are of degree $\alpha_{i}$; notice that the degree of $U^{*}$ is $\alpha=r$, i.e., the degree $r$ of $\delta Q_{r e v}$ and the weight $\alpha$ of $U^{*}$ have to coincide in every case. The Euler vector field is

$$
D=\alpha U^{*} \frac{\partial}{\partial U^{*}}+\sum_{i} \alpha_{i} X^{i *} \frac{\partial}{\partial X^{i *}} .
$$

An integrating factor for $\delta Q_{r e v}$ is given by

$$
f^{*}=\alpha U^{*}-\sum_{i} \alpha_{i} \xi_{i}^{*} X^{i *}
$$

and it is assumed that $f^{*} \not \equiv 0$ as in standard thermodynamics and, moreover, it is assumed that $f^{*} \geq 0$, which is related to the positivity of the absolute temperature. Then, it is possible to show that, as in standard thermodynamics, the potential $\hat{S}^{*}$, which is not quasi-homogeneous, is associated with a positive definite potential $S^{*}$ which is a quasihomogeneous function of degree one with respect to the Euler vector field $D$ :

$$
\hat{S}^{*}-\hat{S}_{0}^{*}=\int_{\Gamma} \frac{\omega}{f^{*}}=\log \left(\frac{S^{*}}{S_{0}^{*}}\right),
$$

The proof is found in Appendix A.

Before continuing our analysis, we recall that a detailed and important study on quasi-homogeneous functions and their application to scaling and universality is the subject of Ref. $[9,10]$ [therein, quasi-homogeneous functions are called "generalized homogeneous functions" , which is a better denomination, but we adopt a common mathematical denomination]. See also [11]. Further mathematical properties are found in Ref. [12] [note that, therein, quasihomogeneous functions are called "almost-homogeneous functions" ( [12], p. 231), whereas the definition "generalized homogeneous functions" is assigned to a further generalization of the equation defining homogeneous and almosthomogeneous functions ( [12], p. 304)].

\section{A. metrical entropy}

By analogy with the construction for standard thermodynamics, a possibility is that $S^{*}$ is the metrical entropy for the system. This is what happens in standard homogeneous thermodynamics and also in black hole thermodynamics, 
and we conjecture that this occurrence is not special. See however the discussion at the end of this section.

We define $T^{*} \geq 0$ by means of

$$
\frac{\partial S^{*}}{\partial U^{*}} \equiv \frac{1}{T^{*}}>0
$$

which means that $S^{*}$ is assumed to be monotonically increasing in $U^{*} . T^{*}$ is another integrating factor for (15), it is such that

$$
\delta Q_{r e v}=T^{*} d S^{*}
$$

$T^{*}$ is a quasi-homogeneous function of degree $r-1$ [see also theorem 1 of [9], where it is shown that the partial derivative $\partial g / \partial X$ of a quasi-homogeneous function $g$ of degree $a$ with respect to a variable $X$ of weight $b$ is a quasihomogeneous function of degree $a-b$ and the same type as $g$ ]. When $r \neq 1$, the function $T$ is not intensive, but changes under quasi-homogeneous rescalings of the system. In the case of a Kerr-Newman black hole, one finds e.g. that, by doubling the mass and the charge, and by quadruplicating the angular momentum, the temperature becomes one half the temperature of the original black hole state. This behavior is well-justified in the light of the Hawking effect.

As a consequence of (19) and of (18), one finds

$$
\frac{\partial \hat{S}^{*}}{\partial U^{*}}=\frac{1}{f^{*}}=\frac{1}{S^{*}} \frac{\partial S^{*}}{\partial U^{*}}=\frac{1}{T^{*} S^{*}},
$$

thus

$$
f^{*}=T^{*} S^{*}
$$

as in standard thermodynamics. From (20) it is easy to show that the quasi-homogeneous entropy can be written as

$$
S^{*}=\alpha \frac{U^{*}}{T^{*}}-\sum_{i} \alpha_{i} \frac{\xi^{*}}{T^{*}} X^{i *} .
$$

In fact, $T^{*}$ is an integrating factor of degree $r-1$ for $\delta Q_{r e v}$, and $d S^{*} \equiv \delta Q_{r e v} / T^{*}$ is an exact quasi-homogeneous Pfaffian form of degree one. Then, as a consequence of lemma 1 in Appendix A (cf. also result 1 therein), one finds $S^{*}=i_{D}\left(\delta Q_{r e v} / T^{*}\right)$, i.e. (23) holds. When all the weights in (23) are equal to one, the well-known expression for the homogeneous thermodynamic entropy is recovered.

\section{B. thermodynamic foliation}

As in the case of standard thermodynamics, one can require that the thermodynamic foliation is defined by the leaves $\hat{S}^{*}=$ const. everywhere in the thermodynamic manifold, except maybe on the boundary. Singularities for $\hat{S}^{*}$ can occur where $f^{*}=0$, i.e., in the set $Z\left(f^{*}\right)=Z\left(T^{*}\right) \cup Z\left(S^{*}\right)$.

The surface $T^{*}=0$ is expected to represent an adiabatic boundary of the thermodynamic manifold. Notice that, as in the case of standard thermodynamics, the set $Z\left(S^{*}\right)$ of zeroes of the function $S^{*}$, if non-empty, is assumed to be contained in the set $Z\left(T^{*}\right)$. In standard thermodynamics the occurrence of a zero for $S$ at a temperature $T>0$ can be considered pathological, a system in such a state should necessarily absorb heat in a neighborhood of this state, whichever reversible process could be considered. Cf. [1]. The same would happen in the quasi-homogeneous case.

Because of (18), the singular values for the thermodynamic foliation are represented by the points where $S^{*}=0$ (if any).

1. a stronger assumption on the domain

One could also assume that

$$
f^{*} \geq 0 \Longleftrightarrow U^{*} \geq b\left(X^{1 *}, \ldots, X^{n *}\right)
$$

where $b\left(X^{1 *}, \ldots, X^{n *}\right)$ is a quasi-homogeneous function of degree $\alpha$ and weights $\left(\alpha_{1}, \ldots, \alpha_{n}\right)$. This function $b\left(X^{1 *}, \ldots, X^{n *}\right)$ plays the role of lowest energy for the system, because by definition $U^{*} \geq b$. The domain $\mathcal{D}$ has to include the following set: 


$$
\operatorname{epi}(b) \equiv\left\{\left(U^{*}, X^{1 *}, \ldots, X^{n *}\right) \mid\left(X^{1 *}, \ldots, X^{n *}\right) \in \mathcal{K}_{(n)}, \quad U^{*} \geq b\left(X^{1 *}, \ldots, X^{n *}\right)\right\}
$$

where $\mathcal{K}_{(n)} \subseteq \mathbb{R}^{n}$ is an open connected set. epi $(b)$ is by definition the epigraph of the function $b$. If $\mathcal{K}_{(n)}$ is closed under quasi-homogeneous dilatations $\left(X^{1 *}, \ldots, X^{n *}\right) \mapsto\left(\lambda^{\alpha_{1}} X^{1 *}, \ldots, \lambda^{\alpha_{n}} X^{n *}\right)$, then epi $(b)$ is closed under quasihomogeneous dilatations involving also $U^{*}$, because $b\left(\lambda^{\alpha_{1}} X^{1 *}, \ldots, \lambda^{\alpha_{n}} X^{n *}\right)=\lambda^{\alpha} b\left(X^{1 *}, \ldots, X^{n *}\right) \leq \lambda^{\alpha} U^{*}$. Moreover, if $\mathcal{K}_{(n)}$ is also closed under the sum and if $b$ is subadditive, then epi(b) is closed under the sum too, thus the closure of $\mathcal{K}_{(n)}$ under quasi-homogeneous dilatations and under the sum allows us to choose $\mathcal{D}=$ epi $(b)$, in analogy with the standard homogeneous case. [Notice that if e.g. $\mathcal{K}_{(n)}=\mathbb{R}_{+}^{n}$, then it is a convex cone which is invariant under quasi-homogeneous dilatations]. An example where the domain $\mathcal{D}$ coincides with the epigraph of a quasi-homogeneous function is furnished by black hole thermodynamics.

Under this assumption on the structure of the domain, one finds that it is not possible to find a state having $S^{*}=0$ at $T^{*}>0$, because by introducing

$$
B^{*} \equiv U^{*}-b\left(X^{1 *}, \ldots, X^{n *}\right) \geq 0
$$

one finds

$$
S^{*}\left(B^{*}, X^{1 *}, \ldots, X^{n *}\right)=S^{*}\left(0, X^{1 *}, \ldots, X^{n *}\right)+\int_{0}^{B^{*}} d Y \frac{1}{T^{*}\left(Y, X^{1 *}, \ldots, X^{n *}\right)}
$$

which cannot be zero for any $B^{*}>0$ (in the last formula mathematical conditions ensuring a continuous entropy at $T^{*}=0$ have been implicitly assumed. Cf. [1]).

As far as the reference state is concerned, we assume that states like the one where all the variables $U^{*}, X^{1 *}, \ldots, X^{n *}$ are zero, and any states which imply the absence of the system, are unphysical. The thermodynamic description starts being meaningful if a statistically relevant number of degrees of freedom is available. Cf. sect. II of paper [1].

\section{C. possible ambiguities}

One may wonder what should happen if one finds that $\omega$ is quasi-homogeneous but the exact weights are not a priori known. In particular, one can assume that their ratio is known, i.e., $\alpha / \alpha_{i}$ and $\alpha_{j} / \alpha_{i}$ are known for all $i \neq j$; then, there is an overall undetermined multiplicative constant $q \neq 0$, in the sense that these ratios don't change if

one multiplies all the weights by the same constant. One could e.g. know $r^{(1)} \equiv \alpha / \alpha_{1}$ and $r_{j}^{(1)} \equiv \alpha_{j} / \alpha_{1}$ for $\alpha_{1} \neq 0$ and for all $j \neq 1$, but the absolute weight $\alpha_{1}$ is not known, thus the weights are $\left(\alpha_{1} r^{(1)}, \alpha_{1}, \alpha_{1} r_{2}^{(1)}, \ldots, \alpha_{1} r_{n}^{(1)}\right)$. An example where this ambiguity appears is represented by black hole thermodynamics (see sect. IV). One can also assign the weights with respect to a scale $\lambda$ whose absolute weight could be unknown. The absolute weights are then of the form $\left(q \alpha, q \alpha_{1}, \ldots, q \alpha_{n}\right)$ with $q$ undetermined. We treat the latter case without loss of generality, being the former equivalent to it under the map $\alpha_{1} \mapsto q \alpha_{1}, q \alpha_{1} r^{(1)} \mapsto q \alpha, q \alpha_{1} r_{i}^{(1)} \mapsto q \alpha_{i}$ for all $i \neq 1$. As a consequence of the undetermined overall factor $q$, one has a one-parameter family of Euler vector fields $\left\{D_{(q)}\right\}$, with

$$
D_{(q)}=q \alpha U^{*} \frac{\partial}{\partial U^{*}}+\sum_{i} q \alpha_{i} X^{i *} \frac{\partial}{\partial X^{i *}} .
$$

Thus, there exist a one-parameter family $\left\{f_{(q)}^{*}\right\}$ of integrating factors, with $f_{(q)}^{*}=\delta Q_{r e v}\left(D_{(q)}\right)$, and a one-parameter family $\left\{\hat{S}_{(q)}\right\}$ of potentials such that

$$
\frac{\delta Q_{r e v}}{f_{(q)}^{*}}=d \hat{S}_{(q)}
$$

and a one-parameter family of entropies $\left\{S_{(q)}^{*}\right\}$ satisfying

$$
\begin{gathered}
d \hat{S}_{(q)}=\frac{d S_{(q)}^{*}}{S_{(q)}^{*}} \\
D_{(q)} S_{(q)}^{*}=S_{(q)}^{*} .
\end{gathered}
$$


The true metrical entropy $S^{*}$ belongs to the family $\left\{S_{(q)}^{*}\right\}$ but is undetermined because the overall factor $q$ is undetermined. In order to see which relation occurs between the various entropies in this family, let us consider a fixed value $\bar{q}$ of the parameter $q$. Then we obtain the entropy $S_{(\bar{q})}^{*}$. If one considers a generic $\hat{q} \neq \bar{q}$, one has

$$
D_{(\hat{q})}=\frac{\hat{q}}{\bar{q}} D_{(\bar{q})}
$$

and

$$
f_{(\hat{q})}^{*}=\frac{\hat{q}}{\bar{q}} f_{(\bar{q})}^{*} .
$$

Then,

$$
\begin{aligned}
& \frac{\delta Q_{\text {rev }}}{f_{(\hat{q})}^{*}}=\frac{\bar{q}}{\hat{q}} \frac{\delta Q_{\text {rev }}}{f_{(\bar{q})}^{*}} \\
& \Longleftrightarrow \\
& d \log \left(S_{(\hat{q})}^{*}\right)=\frac{\bar{q}}{\hat{q}} d \log \left(S_{(\bar{q})}^{*}\right) .
\end{aligned}
$$

As a consequence, by assuming in the integral of $\delta Q_{r e v} / f$ the same reference state $\left(U_{0}^{*}, X_{0}^{1 *}, \ldots, X_{0}^{n *}\right)$, one finds

$$
S_{(\hat{q})}^{*}\left(U^{*}, X^{1 *}, \ldots, X^{n *}\right)=\frac{S_{(\hat{q})}^{*}\left(U_{0}^{*}, X_{0}^{1 *}, \ldots, X_{0}^{n *}\right)}{\left(S_{(\bar{q})}^{*}\left(U_{0}^{*}, X_{0}^{1 *}, \ldots, X_{0}^{n *}\right)\right)^{\bar{q} / \hat{q}}}\left(S_{(\bar{q})}^{*}\left(U^{*}, X^{1 *}, \ldots, X^{n *}\right)\right)^{\bar{q} / \hat{q}}
$$

i.e.

$$
S_{(\hat{q})}^{*}=\zeta_{\hat{q}, \bar{q}}\left(S_{(\bar{q})}^{*}\right)^{\bar{q} / \hat{q}}
$$

where $\zeta_{\hat{q}, \bar{q}} \equiv S_{(\hat{q})}^{*}\left(U_{0}^{*}, X_{0}^{1 *}, \ldots, X_{0}^{n *}\right) /\left(S_{(\bar{q})}^{*}\left(U_{0}^{*}, X_{0}^{1 *}, \ldots, X_{0}^{n *}\right)\right)^{\bar{q} / \hat{q}}$ is a constant, once one has fixed the reference state. The metrical entropy $S^{*}$ is related with the entropy $S_{(\bar{q})}^{*}$ by a simple power law. Cf. also Appendix A.

The one-parameter family of temperatures $\left\{T_{(q)}^{*}\right\}$, with

$$
\frac{1}{T_{(q)}^{*}} \equiv \frac{\partial S_{(q)}^{*}}{\partial U^{*}}
$$

is such that

$$
\frac{1}{T_{(\hat{q})}^{*}}=\zeta_{\hat{q}, \bar{q}} \frac{\bar{q}}{\hat{q}}\left(S_{(\bar{q})}^{*}\right)^{\bar{q} / \hat{q}-1} \frac{1}{T_{(\bar{q})}^{*}} .
$$

Analogous relations exist for the other partial derivatives of $S_{(\bar{q})}^{*}$ and $S_{(\hat{q})}^{*}$. Notice that it holds $f_{(q)}^{*}=T_{(q)}^{*} S_{(q)}^{*}$, because

$$
\frac{\partial \hat{S}_{(q)}^{*}}{\partial U^{*}}=\frac{1}{f_{(q)}^{*}}=\frac{1}{S_{(q)}^{*}} \frac{\partial S_{(q)}^{*}}{\partial U^{*}}=\frac{1}{T_{(q)}^{*} S_{(q)}^{*}} .
$$

\section{1. phenomenological resolution of the ambiguity}

The metrical entropy $S^{*}$ could be phenomenologically identified by means of an absolute temperature thermometer, made of standard matter, allowing to find, as the parameters $U^{*}, X^{1 *}, \ldots, X^{n *}$ are varied, the function $T^{*}$ describing the absolute temperature of the system. As a consequence, also $S^{*}$ can be recovered. We remark that the resolution of the above ambiguity by means of a phenomenological input is completely in the spirit of thermodynamics, which has a phenomenological nature as far as statistical mechanics is not taken into account. 


\section{2. how to fix the absolute weights by means of statistical mechanics}

A priori, even in statistical mechanics, there is a scaling ambiguity, unless the absolute weights are somehow fixed. On this topic, see also sect. XII. In fact, let us assume that the statistical mechanical entropy [calculated by means of some sort of thermodynamic limit or by means of some mean field approximation; cf. sect. XII] under rescaling by means of a scale $\lambda$ is a quasi-homogeneous function of degree $\alpha_{S}$ and weights $\alpha_{1}, \ldots, \alpha_{n}$. This means that $S_{s m}\left(\lambda^{\alpha} U, \lambda^{\alpha_{1}} X^{1}, \ldots, \lambda^{\alpha_{n}} X^{n}\right)=\lambda^{\alpha_{S}} S_{s m}\left(U, X^{1}, \ldots, X^{n}\right)$. By changing the scale $\lambda=\bar{\lambda}^{q}$, the scaling of the variables becomes $\bar{\lambda}^{q \alpha_{i}}$. Thus, there is an overall factor $q$ of ambiguity. Such a redefinition of the scale, unless the absolute weights are somehow fixed, allows to re-map any quasi-homogeneous function of degree $\alpha \neq 0$ into a quasi-homogeneous function of degree one, as noticed in [10]. The statistical mechanical entropy is then assumed to satisfy

$$
S_{s m}\left(\lambda^{q \alpha} U, \lambda^{q \alpha_{1}} X^{1}, \ldots, \lambda^{q \alpha_{n}} X^{n}\right)=\lambda^{q \alpha_{S}} S_{s m}\left(U, X^{1}, \ldots, X^{n}\right) .
$$

where the overall factor ambiguity is enhanced. With respect to the Euler vector field $D_{(q)}$ which corresponds to the above weights it holds $D_{(q)} S_{s m}=q \alpha_{S} S_{s m}$.

We know that from a thermodynamic point of view there is a one-parameter family of possible metrical entropies, each of which is of degree one. The statistical mechanical entropy $S_{s m}$ has to coincide with the metrical thermodynamic entropy $S^{*}$ and it has to hold $T d S_{s m}=T^{*} d S^{*}=\delta Q_{r e v}$. As a consequence, the statistical mechanical entropy has to be a degree one quasi-homogeneous function. This requirement fixes $q \alpha_{S}=1$, and fixes unambiguously the weights [then, it eliminates also the ambiguity in the one-parameter family of thermodynamic entropies].

We could also relax our conjecture about the identification of $S^{*}$ in formula (18) as the metrical entropy. The Clausius-like formula (18) gives in general an empirical entropy which is to be uniquely related with the metrical entropy by identifying the absolute temperature scale as in the standard procedure. One could still assume that the metrical entropy is quasi-homogeneous, but it would be possible in line of principle to require that its degree is $q$, with $q$ non-necessarily equal to one. Then, even in the case where all the weights are known, one would find $S_{\text {metric }}^{*} \propto\left(S^{*}\right)^{q}$, where $S^{*}$ is obtained from formula (18). Cf. also Appendix A. The above phenomenological procedure could be still allowed. Cf. also sect. XII and the conclusions herein.

A further consideration is in order, and it concerns the possible presence of variables of weight zero. All the mathematical approach developed in this section remains unaltered. It is to be noted that, if a variable $I^{*}$ having weight zero is present, the term $I^{*} \partial / I^{*}$ does not appear in the Euler operator and in the integrating factor $f$ the term proportional to $I^{*}$ is missing.

Notice that it is possible that the thermodynamic description allows a reducing/enlarging of the thermodynamic space as the one discussed in Ref. [1]. This means that part of the variables $X^{i *}$ appearing in $\delta Q_{\text {rev }}$ and in $S^{*}$ could be put equal to zero consistently, which means that a meaningful thermodynamic description is still allowed when $X^{i *}=0$ [in standard thermodynamics such variables play e.g. the role of external fields [1]]; Consistency requires that, if $X^{i *}=0$ is allowed, then $\xi_{i}^{*}=0$ as $X^{i *}=0$ [1]. Black hole thermodynamics represents a good example, and this topic is discussed in sect. XI.

We underline that this kind of generalization of thermodynamics can be considered as a sub-case of a generalization proposed by P.T.Landsberg in Ref. [13]. Therein, a discussion of generalized ideal gases appears, where the properties of the following generalized entropy are analyzed:

$$
S=b N \log \left(a \frac{U V^{g}}{N^{h}}\right)
$$

Given the Euler field

$$
D_{\alpha \beta \gamma}=\alpha U \frac{\partial}{\partial U}+\beta V \frac{\partial}{\partial V}+\gamma N \frac{\partial}{\partial N}
$$

it is easy to show that $S$ in (42) is quasi-homogeneous (of degree $\gamma$ ) if and only if $\alpha+g \beta-h \gamma=0$ If the latter constraint is not implemented, then $S$ is not quasi-homogeneous. Homogeneity requires $h=g+1$. 


\section{THE BLACK HOLE CASE}

We summarize herein the results obtained in Ref. [14] in the black hole case (we put $\hbar=c=G=k_{b}=1$, where $k_{b}$ is the Boltzmann constant; moreover, we work with unrationalized electrical units). For a general discussion about black hole thermodynamics see e.g. $[15,16]$. Herein, as well as in sect. V and in sect. VI, we do not use the asterisk, which is in general used only in theoretical sections. The Pfaffian form of black hole thermodynamics for black holes of the Kerr-Newman family is

$$
\delta Q_{r e v} \equiv d M-\Phi d Q-\Omega d J,
$$

where the parameters $M, Q, J$ are the mass $M$, the angular momentum $J$ and the charge $Q$ of the black hole; the angular velocity

$$
\Omega=\frac{J}{M} \frac{1}{2 M^{2}-Q^{2}+2 M \sqrt{M^{2}-Q^{2}-J^{2} / M^{2}}}
$$

and the electric potential

$$
\Phi=\frac{Q\left(M+\sqrt{M^{2}-Q^{2}-J^{2} / M^{2}}\right)}{2 M^{2}-Q^{2}+2 M \sqrt{M^{2}-Q^{2}-J^{2} / M^{2}}}
$$

are associated with the black hole horizon. $M$ in (44) plays the role of internal energy, the remaining terms in (44) being "standard" work terms. Each black hole of the Kerr-Newman family is a stationary solution of the Einstein equations, and it can be considered, from the thermodynamic point of view we develop here, rather loosely speaking, as an "equilibrium state of the geometry". The variation occurring in (44) is taken along such stationary solutions. The thermodynamic domain for the non-extremal black holes is the (open) non-extremal manifold implicitly defined by the inequality $M^{4}-M^{2} Q^{2}-J^{2}>0$; the extremal sub-manifold $M^{4}-M^{2} Q^{2}-J^{2}=0$ is a boundary of the former, and its status is analyzed in Ref. [17] (therein a discussion of the third law of thermodynamics also in the light of this approach is found. We limit ourselves to recall that in black hole thermodynamics the entropic form of the third law is violated [18] but still the unattainability of $T=0$ holds under suitable hypotheses).

The Pfaffian form (44) is integrable, it satisfies the condition $\delta Q_{\text {rev }} \wedge d\left(\delta Q_{\text {rev }}\right)=0$, i.e.

$$
-\partial_{J} \Phi+\partial_{Q} \Omega+\Phi \partial_{M} \Omega-\Omega \partial_{M} \Phi=0 .
$$

Moreover, the Pfaffian form (44) is quasi-homogeneous. In fact, under $M \rightarrow \lambda^{\alpha} M ; Q \rightarrow \lambda^{\alpha} Q ; J \rightarrow \lambda^{2 \alpha} J$, one obtains $\delta Q_{r e v} \rightarrow \lambda^{\alpha} \delta Q_{\text {rev }}$, where $\lambda>0$. The weights $\alpha, \alpha, 2 \alpha$ are not known, only their ratio is determined. Then, the degree of $\delta Q_{\text {rev }}$ is not fixed [14]. Nevertheless, a thermodynamic construction is allowed. One finds that the integrating factor for $\delta Q_{r e v}$ is given by $f=\alpha(M-\Phi Q-2 \Omega J)$, that is

$$
f=\alpha \sqrt{M^{2}-Q^{2}-J^{2} / M^{2}} ;
$$

[it is interesting to notice that this integrating factor is proportional to the horizon coordinate $c$ introduced by B.Carter in [19]. Thus, it is constant on the horizon]. Then, one gets [14]

$$
\int \frac{\delta Q_{r e v}}{f} \equiv \hat{S}-\hat{S}_{0}=\frac{1}{2 \alpha} \log \left(\frac{A}{A_{0}}\right)
$$

where $A=4 \pi\left(M^{2}\left(1+\sqrt{1-Q^{2} / M^{2}-J^{2} / M^{4}}\right)^{2}+J^{2} / M^{2}\right)$ is the black hole area and is positive definite. The main difficulty is in finding the metrical entropy, because of the undetermined overall multiplicative constant $\alpha$. Both $\alpha=1 / 2$ and a undetermined proportionality constant $c_{0}$ appearing in the Bekenstein-Hawking law $S=c_{0} A$ are recovered e.g. by comparing the temperature of the black hole with the Hawking temperature, and one finds $c_{0}=1 / 4$; also the Euclidean path integral method can be used with this aim [20] (see also the papers collected in [21]), as well as a phenomenological plot of the temperature as a function of $M, Q, J$. [Moreover, we notice that the same result $S \propto A$ could be obtained by requiring that the metrical entropy is quasi-homogeneous of degree one with respect to the Euler field $D$ which is obtained by normalizing to one the biggest weight $2 \alpha$ :

$$
D=\frac{1}{2} M \frac{\partial}{\partial M}+\frac{1}{2} Q \frac{\partial}{\partial Q}+J \frac{\partial}{\partial J}
$$


But this normalization rule should be justified on a general thermodynamic footing, and the case $J=0$ (ReissnerNordström solution), being homogeneous in $M, Q$, shows that the physically correct value $\alpha=1 / 2$ cannot be found on the ground of such a rule].

$S$ is quasi-homogeneous of degree one and of type $(1 / 2,1 / 2,1)$ and it is well defined everywhere in the thermodynamic manifold. It is such that $S=0$ only for $M=Q=J=0$, which should not be considered as a state belonging to the thermodynamic manifold [see the discussion in section III; moreover, near $M=0$ quantum gravity effects are non-negligible and General Relativity is expected to be non-viable]. Thus, $Z(S)=\emptyset$ and one finds $Z(f)=Z(T)$, and $\hat{S}$ is regular everywhere; as a consequence, $\hat{S}$ and $S$ could be continued even on the extremal submanifold (but see [17] for arguments against this continuation). All non-extremal states have $T>0$, whereas the extremal boundary corresponds to $T=0$. As it is well-known, one obtains an entropy which is not concave, but simply strictly superadditive when the merging of two black holes is considered. This ensures the second law of thermodynamics [13,22,23]. See also sect. VII.

By introducing

$$
b(Q, J) \equiv \frac{1}{\sqrt{2}} \sqrt{Q^{2}+\sqrt{Q^{4}+4 J^{2}}}
$$

which is a quasi-homogeneous function of degree $1 / 2$ and weights $(1 / 2,1)$, as it can be easily verified (see also [17]), the domain can be also written as follows:

$$
\mathcal{D}=\left\{(M, Q, J) \mid(Q, J) \in \mathbb{R}^{2}, \quad M \geq b(Q, J)\right\} \equiv \operatorname{epi}(b) .
$$

In fact, $M=b(Q, J)$ is the physical [i.e. having positive mass] solution of $M^{4}-M^{2} Q^{2}-J^{2}=0$ and the black hole manifold is $M^{4}-M^{2} Q^{2}-J^{2} \geq 0$, which means $M \geq b$. The function $b(Q, J)$ indicates, for given values of $Q, J$, which is the lowest mass such that a black hole solution can exist. The lowest mass coincides with the mass of the extremal black hole having $Q, J$ as charge and angular momentum respectively.

We point out that the quasi-homogeneous behavior of black hole thermodynamics is not a special feature of the Kerr-Newman family. In fact, it can be realized easily e.g. in the case of the Kerr-Newman-Anti-De-Sitter (KN-AdS) case [24]. A peculiar feature arises, one has to define a thermodynamic angular velocity $\Omega$ as the difference between the angular velocity of the horizon and the angular velocity at infinity, and the same is true in the case of the electric potential [24]. Nevertheless, these definitions are necessary, e.g. the "electric" work term has to involve the difference between the potential at the horizon and the potential at infinity when the latter is not zero, and, on the same footing, the redefinition of $\Omega$ is due to the fact that the angular velocity is not zero at infinity. The change in the energy associated with these work terms has to involve such a difference, which does not appear in the KN case because of the vanishing of $\Phi$ and of $\Omega$ at infinity.

General Relativity is surprising from a thermodynamic point of view. Black hole solutions of the Einstein equations are involved with an integrability condition (47), and they allow a fine explicit thermodynamic construction à la Carathéodory. One finds a link with thermodynamics which is a priori unexpected and it is not simply the formal analogy between laws of thermodynamics and laws of black hole mechanics, because the above construction belongs to the thermodynamic framework, apart from the geometric inputs $\Omega, \Phi$, which come from General Relativity, and a comparison with the Hawking effect, which fixes $\alpha=1 / 2$. See also the discussion in Ref. [14].

\section{NEWTONIAN GRAVITY. A MODEL OF HERTEL, NARNHOFER AND THIRRING}

There exists an interesting statistical mechanical calculation for gravitating fermions which corroborates the idea that, in presence of gravity, a quasi-homogeneous thermodynamics is allowed [25-27]. The model (HNT model in the following) involves non-relativistic fermions interacting by means of a Coulomb potential and a Newtonian potential, with vanishing [or small] total charge. Thus, the calculation does not involve General Relativity but only Newtonian gravity. Nevertheless, the result is still interesting and, to some extent, puzzling. The quantum mechanical Hamiltonian which is considered is

$$
H=\sum_{i=1}^{N} \frac{\vec{p}_{i}^{2}}{2 M_{i}}+\sum_{i<j} \frac{e_{i} e_{j}-G m_{i} m_{j}}{\left|\vec{x}_{i}-\vec{x}_{j}\right|}
$$


The authors state that a thermodynamic limit exists if the following asymptotic behavior is allowed for $U, V: V \sim 1 / N$, $U \sim N^{7 / 3}$, and the micro-canonical entropy is of degree one in the number $N$ of fermions. The scaling properties of $U, V$ with $N$ are peculiar, they are homogeneous of degree one if the potential term in (53) is suppressed by means of a multiplicative factor $1 / N^{2 / 3}$, which means that, as the system gets larger, the interaction becomes weaker [27]. If this suppression is not in agreement with the physics, and if the fermions do not become relativistic, then the micro-canonical entropy $S(U, V, N)$ is a quasi-homogeneous function of degree one, and the weights of $U, V, N$ are $7 / 3,-1,1$ respectively. Cf. also sect. XII, where the notion of quasi-homogeneous thermodynamic limit is introduced. The Euler field is

$$
D_{U V N}=\frac{7}{3} U \frac{\partial}{\partial U}-V \frac{\partial}{\partial V}+N \frac{\partial}{\partial N} .
$$

For details of the highly non-trivial calculations, see Refs. $[25,26,28]$. We are interested here in pointing out that, as stressed in Ref. [25], there is a one-parameter family of equivalent limits $N \rightarrow \infty$, whose parameter $\gamma$ remains fixed by the requirement that the ground state energy goes like $N^{7 / 3}$, according to a result of Ref. [29]. A scaling ambiguity, which would reflect itself in the undetermined weights $(-2 \gamma+5 / 3,3 \gamma, 1)$ of $(U, V, N)$ respectively, is thus solved by comparing with the scaling behavior of the ground state. See also [28]. Another important point is that the temperature-dependent Thomas-Fermi equation, which allows these scaling properties, becomes exact in the thermodynamic limit for the system under discussion [25].

We limit ourselves to underline that Newtonian gravity furnishes a statistical mechanical example where unusual scaling laws for the "extensive" variables are allowed. The purely attractive nature of gravity plays a major role, because it does not saturate, i.e., it does not allow to obtain a ground state proportional to the number of particles [29]. Notice also that a lack of concavity is allowed [27].

It is interesting to notice that another model involving different scaling relations has been developed in Ref. [30]. Therein, a classical gas of non-relativistic particles which interact by means of Newtonian gravity is considered in a diluted regime where particles of mass $m$ are enclosed in a box; the following behavior is recovered in a non-conventional thermodynamic limit where $V / N^{3} \rightarrow$ const. as $N \rightarrow \infty$. A dilution parameter

$$
\xi \equiv \frac{1}{G m^{2}} \frac{U V^{1 / 3}}{N^{2}}
$$

is kept constant in the thermodynamic limit. One finds that $S(U, V, N)$ is quasi-homogeneous of degree one and weights $(1,3,1)$, as can be inferred from Ref. [30]. Cf. also sect. XII. This model differs from the previous one because it involves classical matter, the gas is kept diluted in the thermodynamic limit, no collapse is considered. Note that even the HNT model can be related with the parameter $\xi$ introduced in (55), in fact even in HNT model this parameter is constant in the appropriate thermodynamic limit. $\xi$ can be qualitatively interpreted as the ratio between the internal energy and the Newtonian gravitational energy, in fact the gravitational energy of a homogeneous sphere of mass $M \equiv N^{2} m$ is proportional to $G M^{2} / V^{1 / 3}$. Thus, in both cases, the requirement of a non-negligible contribution of the gravitational energy to the internal energy is realized in the thermodynamic limit. In the HNT model, a self-bounded system is considered, like a (cold) star, where $V$ contracts as the mass is increased; in the model of Ref. [30], the physical conditions are different, a gaseous system is considered, the energy per particle remains constant and small, and the systems remains gaseous in the thermodynamic limit.

In the regime where gravitational forces cannot be neglected, one can expect that a different kind of quasi-homogeneous thermodynamics can arise, with different weights, depending on the actual physical conditions, e.g., the amount and the type of matter. Semi-relativistic matter is expected to behave in a different manner (cf. Ref. [29]). The point is that a different scaling behavior of the ground-state of the matter can be expected if relativistic effects in the kinetic energy are non-negligible. A self-consistent calculation taking into account General Relativity is expected to give another kind of contribution. An approach, combining in a self-consistent way general-relativistic equations and finite-temperature Thomas-Fermi approximation for the thermodynamic functionals would be required. In this direction, see Ref. [31]. It is also interesting to point out that such an approach probably would not be useful for understanding black hole thermodynamics, in fact no stable ground state for the matter could exist by hypothesis (a collapse of the matter beyond the possible stable states represented by white dwarf and neutron stars should take place, with no possibility to prevent the formation of an event horizon).

\section{A LOOK AT THE THERMAL GEON AND AT SELF-GRAVITATING RADIATION}

We discuss naively the scaling properties of the so-called thermal geons [32]. A geon is a "gravitational electromagnetic entity" which consists in a self-gravitating ensemble of electromagnetic modes [33]. The most stable configuration 
is such that the photons are distributed within a toroidal region of the space-time, but also a spherical distribution can be allowed. Geons don't represent strictly stationary solutions of the Einstein equations, because a leakage of photons to infinity is allowed. Nevertheless, they can be considered as metastable quasi-stationary solutions. Moreover, sizes are considered where no contribution of electron physics, due to pair creation by the electromagnetic field, is considered, and no zero-point energy is taken into account. In Ref. [32] the idea of a geon has been generalized to the case of thermally distributed electromagnetic modes. The main assumptions are the following ones: a) the metric is a spherosymmetric static diagonal metric in the Schwarzschild gauge; b) there exist two separate classes of electromagnetic modes; the first class is constituted by bounded null geodesics which represent modes whose energy cannot escape to infinity [the actual rate of flux to infinity goes to zero exponentially with the ratio between the dimensions of the geon and the wavelength $\lambda$ ]. The second class is constituted by free electromagnetic modes, i.e. by null geodesics which reach infinity. c) The energy of the free modes is zero, the energy of the bounded modes of frequency $\Omega$ is [in natural units]

$$
E_{\Omega}=\Omega \frac{1}{\exp \left(\frac{\Omega}{T}\right)-1}
$$

which is the usual distribution for black body radiation and $T$ is the temperature. See also [32] on this topic. The active region of the geon is defined as the region where the metric coefficient $g_{00}$ satisfies $\left|g_{00}\right|<1$. By defining $R$ as the radius of the active region and $M$ as its mass, the following scaling laws can be deduced [32]:

$$
\begin{gathered}
R \sim R_{T} \equiv\left(\frac{15 \hbar^{3} c^{7}}{8 \pi^{3} G}\right)^{1 / 2} \frac{1}{T^{2}} \propto \frac{1}{T^{2}} \\
M \sim M_{T} \equiv\left(\frac{15 \hbar^{3} c^{11}}{8 \pi^{3} G^{3}}\right)^{1 / 2} \frac{1}{T^{2}} \propto \frac{1}{T^{2}} .
\end{gathered}
$$

As an order of magnitude estimation on the same footing of the previous evaluations, one can see that the entropy behaves as $S \propto V T^{3}$, where $V$ is the volume of the active region, thus $S \propto T^{-3}$. From the above naive considerations, one finds that $S$ is a quasi-homogeneous function

$$
S\left(\lambda^{-2} M, \lambda^{-6} V\right)=\lambda^{-3} S(M, V) ;
$$

In the case of a massless gas, there is an ambiguity in the identification of the absolute weights of the variables. There is an overall multiplicative factor to be determined in the Euler vector field. Let us assume that $S$ is of degree one, as suggested by the formal picture of thermodynamics (cf. sect. III and sect. XII); then $T$ has degree $-1 / 3$ and the scaling law for the fundamental equation is

$$
S\left(\lambda^{2 / 3} M, \lambda^{2} V\right)=\lambda S(M, V),
$$

i.e., $M$ has weight $2 / 3$ and $V$ has weight 2 . We note that the ratio $M / V^{1 / 3}$ is scale-invariant and it is the same ratio which is kept constant in the thermodynamic limit discussed in [30].

The above result about thermal radiation can be confirmed by comparing it with the study of Ref. [34]. Black body self-gravitating radiation enclosed in a spherical box of radius $R$ is considered in [34]. It is shown that the maximization of the entropy in a spherosymmetric geometry leads to the following results: i) the metric is static; ii) the perfect fluid of photons satisfies the Tolman-Oppenheimer-Volkov (TOV) equation. In particular, the TOV equation one obtains is scale-invariant. By introducing the density $\rho(r)$, the mass $m(r)=\int_{0}^{r} d y 4 \pi y^{2} \rho(y)(r$ is the radial coordinate) one can define, by following [34], the dimensionless variables

$$
\begin{aligned}
\mu & =m(r) / r ; \\
q & =d m / d r=4 \pi r^{2} \rho(r) ;
\end{aligned}
$$

and the TOV becomes equivalent to the following couple of equations:

$$
\begin{aligned}
r \frac{d q}{d r} & =\frac{2 q}{1-2 \mu}\left(1-4 \mu-\frac{2}{3} q\right), \\
r \frac{d \mu}{d r} & =q-\mu .
\end{aligned}
$$

No equilibrium is attained if $\mu>0.25$ [34]. We are interested here in the scaling properties of the Pfaffian form 


$$
\delta Q_{\text {rev }}=d M+p d V,
$$

where $M \equiv m(R)$ is the mass, $V$ is the volume (as seen from infinity) and $p=\rho(R) / 3$ is the pressure as a function of the box radius. These variations are to be intended, as in the black hole case, as "on shell" , i.e., along static spherosymmetric equilibrium solutions of the TOV. Each solution represents a thermodynamic equilibrium state. Under the scaling $M \rightarrow \lambda M ; V \rightarrow \lambda^{3} V ; p \rightarrow \lambda^{-2} p$, which corresponds to (30) of [34] and is allowed by the scale invariance of the TOV, one finds $\delta Q_{\text {rev }} \rightarrow \lambda \delta Q_{\text {rev }}$. Particularly, one has

$$
D=\alpha\left(M \frac{\partial}{\partial M}+3 V \frac{\partial}{\partial V}\right),
$$

where $\alpha$ has to be determined. From $d V=4 \pi R^{2} d R$ and from (64) one finds

$$
\delta Q_{r e v}=\frac{4}{3} q d R
$$

and the integrating factor

$$
\delta Q_{\text {rev }}(D)=\alpha(M+3 p V)=\alpha \frac{1}{3}(3 \mu+q) R .
$$

Thus,

$$
\frac{\omega}{f}=\frac{4}{\alpha} \frac{q}{3 \mu+q} \frac{d R}{R} .
$$

By comparing this result with the ratio $d S / S$ which can be obtained by the exact result (34) of [34], one finds that $\alpha=2 / 3$. As the consequence, the weights of $D$ are the same as in the case of thermal geons (notice that the requirement for $S$ to be quasi-homogeneous of degree one in the scale factor $\lambda$ would lead to the scaling $r \rightarrow$ $\lambda^{2 / 3} r ; m \rightarrow \lambda^{2 / 3} m ; \rho \rightarrow \lambda^{-4 / 3} \rho$ in (30) of [34]).

\section{CONSEQUENCES OF THE NON-EXTENSIVITY OF $S$}

We have to underline that the lack of extensivity has important consequences on the properties of the entropy. In fact, if one considers for a continuous entropy $S$ superadditivity (S), concavity (C) and homogeneity $(\mathrm{H})$, one finds:

a) $(\mathrm{H})$ and $(\mathrm{S})$ imply $(\mathrm{C})[35]$;

b) $(\mathrm{H})$ and $(\mathrm{C})$ imply $(\mathrm{S})[36]$;

c) (S), with the condition $S(0)=0$, and (C) imply $(\mathrm{H})[8,22]$.

We recall that a function $h(x)$ of $n$ variables, collectively indicated with $x$ in this section, is superadditive if $h(x+y) \geq h(x)+h(y)$ for all $x, y$ in its domain. Superadditivity, in the case of entropy, means that the principle of entropy increase in a irreversible adiabatic process is respected. Moreover, $(\mathrm{C})$ requires a convex domain, $(\mathrm{S})$ a domain which has to be closed under addition, and $(\mathrm{H})$ a domain which has to be closed under multiplication of $x$ by a real scalar $\lambda>0$ (a cone).

Notice that the condition $S(0)=0$ appearing in c) is a mathematical condition which could be required even if the state $x=0$ does not belong to the thermodynamic space, in the light of our discussion in sect. III; see however also [22], where $x=0$ is included in the thermodynamic space. One could consider an extended domain including $x=0$, on a purely mathematical ground, even if the thermodynamic formalism at such a point is meaningless. Moreover, $S$ is required to be a continuous function. Then, if homogeneity does not hold and $S(0)=0$, either superadditivity or concavity has to be violated. It can be easily shown that the condition $S(0)=0$ in c) can be replaced by the (natural) requirement that $S(x) \geq 0$ for any state $x$ in the thermodynamic domain. In fact, (C) implies

$$
S\left(\frac{1}{2} y+\frac{1}{2} z\right) \geq \frac{1}{2} S(y)+\frac{1}{2} S(z)
$$

for any $y, z ;$ moreover, (S) implies

$$
S(2 x) \geq 2 S(x) .
$$


If $z=0$ and $y=2 x$, from (70) one obtains

$$
S(x) \geq \frac{1}{2} S(2 x)+\frac{1}{2} S(0) .
$$

Then, $1 / 2 S(2 x)+1 / 2 S(0) \leq S(x) \leq 1 / 2 S(2 x)$, which implies $S(0) \leq 0$. As a consequence, the requirement of a nonnegative entropy $S$, together with $(\mathrm{S})$ and $(\mathrm{C})$, implies $S(0)=0$, thus also $(\mathrm{H})$ has to hold. This shows that the condition $S(0)=0$, in the framework of thermodynamics, where $S \geq 0$, cannot be actually considered as a true restriction leaving room for a thermodynamics in which a concave and superadditive but non-homogeneous entropy is allowed if $S$ is defined in $x=0$ [of course, this holds as far as negative values of $S$ are forbidden]. Notice that $S^{*}(0)=0$ for a continuous quasi-homogeneous entropy defined in 0 .

Superadditivity of the entropy means that for the thermodynamic system the entropy does not increase by fragmenting the system [8]. From the point of view of the energy representation, to be discussed in sect. X, superadditivity of the entropy is equivalent to the subadditivity of the energy under the condition (19) [for a proof, see Refs. [35,36]. This equivalence holds even if conditions $(\mathrm{C}),(\mathrm{H})$ are violated]. Subadditivity of the energy means that exploding is not energetically advantageous for the system $[37,38]$. The relevance of $(\mathrm{S})$ for the second law of thermodynamics is discussed and underlined in Ref. [13]. Accordingly, one should privilege the superadditivity property against the concavity, and superadditivity should be required as a fundamental property for the quasi-homogeneous picture of thermodynamics. Notice that a lack of concavity for thermodynamics in presence of gravity is verified in Refs. [39,40], where negative heat capacities in presence of gravity are calculated. Therein, a discussion on how to deal with the lack of the standard stability properties of thermodynamics is sketched. See also [41,42]. It is to be noted that this lack of concavity in presence of gravity forces to abandon the usual Gibbsian scheme for thermodynamics, and the homogeneity property has to be withdrawn because of a), if one adopts the superadditivity as a fundamental property for the metrical entropy of a system. Due to the above equivalence between superadditivity of the entropy and subadditivity of the internal energy, one can naively justify this choice by underlining that, because of the purely attractive nature of gravity, internal energy of a self-gravitating system should be strictly subadditive, in fact energy decreases as the accretion of matter increases. Thus, there is an implosive instability of gravity, which eventually leads to the formation of black holes, to be identified as very long-lived metastable states. By passing, we note that there is another instability which is opposite in character with respect to the one implied by gravity. It involves system fragmentation/explosion because of a superadditive energy [37,38]. It should be characterized only by unstable states [systems with a suitable charge excess would be explosive [28]. See also the works of E.H.Lieb collected in Ref. [43], in particular the review [44]; see also [45]].

\section{SUMMARY OF CONSTRUCTIVE ASSUMPTIONS}

We summarize the set of constructive assumptions upon which our approach is based. They correspond to the assumptions leading to standard homogeneous thermodynamics [1], the difference consists in the substitution of the homogeneity symmetry with the quasi-homogeneity symmetry and also in the explicit request that the entropy is superadditive. We comment only some assumptions which require further remarks with respect to the discussion developed in the text.

a1) The quasi-homogeneity symmetry which characterizes the thermodynamic system is translated into the quasihomogeneity of the Pfaffian form $\delta Q_{r e v}=d U^{*}-\sum_{i=1}^{n} \xi_{i}^{*} d X^{i *}$.

a2) The quasi-homogeneity symmetry of $\delta Q_{r e v}$ is nontrivial.

a3) The thermodynamic foliation is defined by the leaves $\hat{S}^{*-1}(c)$, where $c \in \mathbb{R}$ is a constant, everywhere in $\mathcal{D}$.

This assumption does not leave room for foliations which are based on a quasi-homogeneous $S^{*}$ which is allowed to be negative. Cf. the discussion in [1] for the homogeneous case.

a4) The integrating factor $f^{*}$ is non-negative.

a5) The metrical entropy $S^{*}$ is quasi-homogeneous of degree one.

This assumption could also be weakened, as discussed in the text. 
a6) The metrical entropy $S^{*}$ is superadditive.

This assumption substitutes the requirement for a concave entropy occurring in standard homogeneous thermodynamics. Notice that in standard thermodynamics it would lead again to a concave metrical entropy, because of the implication a) of sect. VII. Cf. [35].

a7) We require that $Z\left(S^{*}\right) \subseteq Z\left(T^{*}\right)$.

This assumption is e.g. implemented as in sect. III B 1.

One can also add the following assumptions which appear to be physically appealing:

a8) We require that to each level set $S^{*}=$ const. corresponds a unique leaf.

This assumption means that two states of the same quasi-homogeneous system lying on the same isoentropic surface are path-connected, i.e. it is possible to find a reversible adiabatic path connecting each other.

a9) We require that $\frac{\partial S^{*}}{\partial V^{*}}$ is positive if $V^{*}$ is the volume of the system.

This simply means that the pressure $p^{*}$ is positive whenever it is definite.

a10) We require that $f^{*}=0$ corresponds to an integral manifold of $\delta Q_{\text {rev }}$.

This assumption is natural and is related to the problem of the third law. Cf. [1] for standard thermodynamics; see also [17] for the black hole case and also Appendix B. 


\section{GENERALIZED GIBBS-DUHEM EQUATION}

Quasi-homogeneity of $S^{*}$ allows to find a generalization of the standard Gibbs-Duhem equation [46].

\section{A. case where none independent variable of weight zero appears}

We first discuss the case where none independent variable of weight zero appears. By differentiating (23) one finds

$$
d S^{*}=\alpha \frac{1}{T^{*}} d U^{*}+\alpha U^{*} d\left(\frac{1}{T^{*}}\right)-\sum_{i} \alpha_{i} \frac{\xi_{i}^{*}}{T^{*}} d X^{i *}-\sum_{i} \alpha_{i} X^{i *} d\left(\frac{\xi_{i}^{*}}{T^{*}}\right)
$$

and by comparing with

$$
d S^{*}=\frac{1}{T^{*}} d U^{*}-\sum_{i} \frac{\xi_{i}^{*}}{T^{*}} d X^{i *}
$$

one finds that the following generalized Gibbs-Duhem equation has to hold:

$$
\alpha U^{*-\frac{\alpha-1}{\alpha}+1} d\left(\frac{U^{* \frac{\alpha-1}{\alpha}}}{T^{*}}\right)-\sum_{i} \alpha_{i} X^{i * \frac{\alpha_{i}-1}{\alpha_{i}}+1} d\left(\frac{X^{i * \frac{\alpha_{i}-1}{\alpha_{i}}} \xi_{i}^{*}}{T^{*}}\right)=0 .
$$

This equation, as in the usual thermodynamic case, allows to express the differential of a would-be intensive variable as a function of all the other ones, and then to find such a would-be intensive variable apart from a integration constant (cf. [46] for the case of standard thermodynamics). In fact, one could e.g. find for $1 / T^{*}$ :

$$
d \log \left(\frac{1}{T^{*}}\right)=-\frac{\alpha-1}{f^{*}} d U^{*}+\frac{1}{f^{*}} \sum_{i} \alpha_{i} X^{i *^{-\frac{\alpha_{i}-1}{\alpha_{i}}+1}} d\left(X^{i * \frac{\alpha_{i}-1}{\alpha_{i}}} \xi_{i}^{*}\right) .
$$

Notice that

$$
d \log \left(\frac{1}{T^{*}}\right)=-d \log \left(f^{*}\right)+\frac{\delta Q_{r e v}}{f^{*}} .
$$

Eqn. (76) is implemented if (18) and (74) hold. Let us consider the inverse problem where one assigns $n$ wouldbe intensive functions $\xi_{1}^{*}, \ldots, \xi_{n}^{*}$ which are quasi-homogeneous in $U^{*}, X^{1 *}, \ldots, X^{n *}$ and such that $\delta Q_{r e v}$ is quasihomogeneous as well. Eqn. (76), or, equivalently, eqn. (77) can be used for recovering $T^{*}$, and then for reconstructing $S^{*}$ by means of (18), if and only if one has ensured that $\delta Q_{r e v} / f^{*}$ is an exact differential, i.e., that $\delta Q_{r e v}$ is integrable and $f^{*}$ is an integrating factor for $\delta Q_{\text {rev }}$. Otherwise, (76) is not even meaningful (a closed form on the left side of (77) should be equal to a non-closed form on the right side of the same equation). Cf. also [1].

Notice that, if $S^{*}$ has degree $q$, then (75) becomes

$$
\frac{\alpha}{q} U^{*-\frac{\alpha-q}{\alpha}+1} d\left(\frac{U^{* \frac{\alpha-q}{\alpha}}}{T^{*}}\right)-\sum_{i} \frac{\alpha_{i}}{q} X^{i * \frac{\alpha_{i}-q}{\alpha_{i}}+1} d\left(\frac{X^{i * \frac{\alpha_{i}-q}{\alpha_{i}}} \xi_{i}^{*}}{T^{*}}\right)=0 .
$$

See also Appendix C.

\section{B. case where independent variables of weight zero appear}

Let us assume that, the independent variables $X^{i *}$ have weight zero for $i=p+1, \ldots, n$, with $p<n$. This means that $\xi_{i}^{*}$ have degree $\alpha$ for $i=p+1, \ldots, n$. The Euler vector field is

$$
D=\alpha U^{*} \frac{\partial}{\partial U^{*}}+\sum_{i \leq p} \alpha_{i} X^{i *} \frac{\partial}{\partial X^{i *}}
$$


and the integrating factor is

$$
f^{*}=\alpha U^{*}-\sum_{i \leq p} \alpha_{i} \xi_{i}^{*} X^{i *}
$$

Then, one has

$$
S^{*}=\frac{1}{T^{*}}\left(\alpha U^{*}-\sum_{i \leq p} \alpha_{i} \xi_{i}^{*} X^{i *}\right)
$$

It is easy to show that the Gibbs-Duhem equation in this case is

$$
\alpha U^{*-\frac{\alpha-1}{\alpha}+1} d\left(\frac{U^{* \frac{\alpha-1}{\alpha}}}{T^{*}}\right)-\sum_{i \leq p} \alpha_{i} X^{i *-\frac{\alpha_{i}-1}{\alpha_{i}}+1} d\left(\frac{X^{i * \frac{\alpha_{i}-1}{\alpha_{i}}} \xi_{i}^{*}}{T^{*}}\right)+\sum_{p<i \leq n} \frac{\xi_{i}^{*}}{T^{*}} d X^{i *}=0 .
$$

One can also obtain the analogous of eqn. (76)

$$
\begin{aligned}
d \log \left(\frac{1}{T^{*}}\right) & =-\frac{\alpha-1}{f^{*}} d U^{*}+\frac{1}{f^{*}} \sum_{i \leq p} \alpha_{i} X^{i * \frac{\alpha_{i}-1}{\alpha_{i}}+1} d\left(X^{i * \frac{\alpha_{i}-1}{\alpha_{i}}} \xi_{i}^{*}\right) \\
& -\frac{1}{f^{*}} \sum_{p<i \leq n} \xi_{i}^{*} d X^{i *} .
\end{aligned}
$$

(77) still holds.

Concerning the Gibbs-Duhem equation in standard thermodynamics, see [47].

\section{C. examples}

In the black hole case, as well known, one has [we don't write the asterisk in what follows]

$$
S=\frac{M}{2 T}-\frac{\Phi Q}{2 T}-\frac{\Omega J}{T}
$$

and

$$
d S=\frac{1}{T} d M-\frac{\Phi}{T} d Q-\frac{\Omega}{T} d J
$$

the generalized Gibbs-Duhem equation, associated with the quasi-homogeneity of $S$, is

$$
-\frac{1}{2 T} d M+\frac{M}{2} d\left(\frac{1}{T}\right)+\frac{\Phi}{2 T} d Q-\frac{Q}{2} d\left(\frac{\Phi}{T}\right)-J d\left(\frac{\Omega}{T}\right)=0,
$$

which can be rewritten as [14]

$$
\frac{1}{2} M^{2} d\left(\frac{1}{M T}\right)-\frac{1}{2} Q^{2} d\left(\frac{\Phi}{Q T}\right)-J d\left(\frac{\Omega}{T}\right)=0
$$

Then, one can find $1 / T$ from

$$
d \log \left(\frac{1}{T}\right)=\frac{1}{f}\left(\frac{1}{2} d M+\frac{1}{2} Q^{2} d\left(\frac{\Phi}{Q}\right)+J d \Omega\right) .
$$

It can be easily shown that $1 / T$ can be determined apart from an undetermined multiplicative constant. 


\section{ENERGY REPRESENTATION AND LEGENDRE TRANSFORM}

In the energy representation, the fundamental equation is $U^{*}=U^{*}\left(S^{*}, X^{1 *}, \ldots, X^{n *}\right)$. One has

$$
d U^{*}=T^{*} d S^{*}+\sum_{i} \xi_{i}^{*} d X^{i *}
$$

$U^{*}$ is a quasi-homogeneous function of degree $r$ and type $\left(1, \alpha_{1}, \ldots, \alpha_{n}\right) ; T^{*}$ is quasi-homogeneous of degree $r-1, \xi_{i}^{*}$ are quasi-homogeneous of degree $\alpha_{i}-r$. (89) represents an exact quasi-homogeneous Pfaffian form of degree $r$. The Euler operator is

$$
D=S^{*} \frac{\partial}{\partial S^{*}}+\sum_{i} \alpha_{i} X^{i *} \frac{\partial}{\partial X^{i *}}
$$

One has then

$$
U^{*}=\frac{1}{r}\left(T^{*} S^{*}+\sum_{i} \alpha_{i} \xi_{i}^{*} X^{i *}\right)
$$

[Notice that the generalization to the case where $S^{*}$ is quasi-homogeneous of degree $q$ is trivial].

As far as the Gibbs-Duhem equation is concerned, one easily finds [we shift to the case where $S^{*}$ has degree $q$ ]

$$
\frac{q}{r} S^{*-\frac{q-r}{q}+1} d\left(S^{* \frac{q-r}{q}} T^{*}\right)+\sum_{i} \frac{\alpha_{i}}{r} X^{i *^{-\frac{\alpha_{i}-r}{\alpha_{i}}+1}} d\left(X^{i * \frac{\alpha_{i}-r}{\alpha_{i}}} \xi_{i}^{*}\right)=0
$$

For a proof see Appendix C. One can e.g. determine $T^{*}$ from

$$
d\left(S^{* \frac{q-r}{q}} T^{*}\right)=S^{* \frac{q-r}{q}-1} \frac{1}{q} \sum_{i} \alpha_{i} X^{i * \frac{\alpha_{i}-r}{\alpha_{i}}+1} d\left(X^{i * \frac{\alpha_{i}-r}{\alpha_{i}}} \xi_{i}^{*}\right) .
$$

A formula analogous to (82) can be obtained if some independent zero-weight variables appear; one obtains (cf. Appendix C)

$$
\frac{q}{r} S^{*-\frac{q-r}{q}+1} d\left(S^{* \frac{q-r}{q}} T^{*}\right)+\sum_{i \leq p} \frac{\alpha_{i}}{r} X^{i *-\frac{\alpha_{i}-r}{\alpha_{i}}+1} d\left(X^{i * \frac{\alpha_{i}-r}{\alpha_{i}}} \xi_{i}^{*}\right)-\sum_{p<i \leq n} \xi_{i}^{*} d X^{i *}=0 .
$$

\section{A. Legendre transforms}

Legendre transforming of the potentials involves the usual procedure. A noticeable difference, with respect to standard thermodynamics, is that, if, between the $n+1$ independent variables, no variable of weight zero appears and no intensive dependent variable occurs, then one can iterate the Legendre transformations of the potential $n+1$ times and find a potential which is not identically vanishing. Moreover, the would-be intensive variables appear in the Euler operator involving the new independent variables. Let us e.g. consider the quasi-homogeneous free energy

$$
F^{*}\left(T^{*}, X^{1 *}, \ldots, X^{n *}\right)=U^{*}-T^{*} S^{*} .
$$

It is a quasi-homogeneous function of degree $r$ with respect to the Euler operator

$$
D_{1}=(r-1) T^{*} \frac{\partial}{\partial T^{*}}+\sum_{i} \alpha_{i} X^{i *} \frac{\partial}{\partial X^{i *}} .
$$

One can Legendre-transform also with respect to the remaining $n$ variables $X^{1 *}, \ldots, X^{n *}$ :

$$
G_{n+1}^{*}\left(T^{*}, \xi_{1}^{*}, \ldots, \xi_{n}^{*}\right)=U^{*}-T^{*} S^{*}-\sum_{i=1}^{n} X^{i *} \xi_{i}^{*} \not \equiv 0 .
$$


The corresponding Euler operator is

$$
D_{n+1}=(r-1) T^{*} \frac{\partial}{\partial T^{*}}+\sum_{i}\left(r-\alpha_{i}\right) \xi_{i}^{*} \frac{\partial}{\partial \xi_{i}^{*}} .
$$

Notice that, all the potentials which are obtained by Legendre-transforming the internal energy $U^{*}$ are quasihomogeneous of degree $r$ with respect to the corresponding Euler operator; all the Massieu-Planck potentials, which are obtained by Legendre-transforming the entropy $S^{*}$, are quasi-homogeneous of degree one. In other terms, the Legendre transform preserves the degree of quasi-homogeneity (but it changes variables and weights: the function

$$
h^{*}\left(\xi^{*}, Y^{*}\right)=g^{*}-\xi^{*} X^{*} .
$$

which is obtained by a Legendre transform with respect to a variable $\xi$ of weight $\alpha$ of a function $g\left(X^{*}, Y^{*}\right)$ which is quasi-homogeneous of degree $r$ and weights $(r-\alpha, r-\beta)$, has weight $(\alpha, r-\beta))$. For a proof, see Ref. [9] (theorem 2, Appendix A therein).

\section{1. case where weight-zero independent variables appear}

Let us consider the case where a variable $\eta^{*}$ of degree $r$ and a variable $I^{*}$ of weight zero appears:

$$
d U^{*}=T^{*} d S^{*}+\xi^{*} d X^{*}+\eta^{*} d I^{*} .
$$

The Euler operator is

$$
D=S^{*} \frac{\partial}{\partial S^{*}}+\beta X^{*} \frac{\partial}{\partial X^{*}} .
$$

One can Legendre-transform three times, obtaining

$$
G_{3}\left(T^{*}, \xi^{*}, \eta^{*}\right)=\frac{1}{r}\left[(1-r) T^{*} S^{*}-(r-\beta) \xi^{*} X^{*}-r \eta^{*} I^{*}\right]
$$

and

$$
D_{3}=(r-1) T^{*} \frac{\partial}{\partial T^{*}}+(r-\beta) \xi^{*} \frac{\partial}{\partial \xi^{*}}+r \eta^{*} \frac{\partial}{\partial \eta^{*}}
$$

2. case with would-be intensive variable of degree zero

Let us consider the case where a variable $\zeta^{*}$ of degree zero and a variable $Z^{*}$ of weight $r$ appears:

$$
d U^{*}=T^{*} d S^{*}+\xi^{*} d X^{*}+\zeta^{*} d Z^{*}
$$

The Euler operator is

$$
D=S^{*} \frac{\partial}{\partial S^{*}}+\beta X^{*} \frac{\partial}{\partial X^{*}}+r \zeta^{*} \frac{\partial}{\partial \zeta^{*}} .
$$

One can Legendre-transform three times, obtaining

$$
G_{3}\left(T^{*}, \xi^{*}, \zeta^{*}\right)=\frac{1}{r}\left[(1-r) T^{*} S^{*}-(r-\beta) \xi^{*} X^{*}\right]
$$

and

$$
D_{3}=(r-1) T^{*} \frac{\partial}{\partial T^{*}}+(r-\beta) \xi^{*} \frac{\partial}{\partial \xi^{*}} .
$$

This case is analogous to what happens in standard thermodynamics e.g. when one passes from $U(S, V, N)$ to $F(T, V, N)$ and then to $G(T, p, N)$. 


\section{B. from the entropy to the energy representation and further considerations}

In order to pass from the entropy representation $S^{*}\left(U^{*}, X^{1 *}, \ldots, X^{n *}\right)$ to the energy representation $U^{*}\left(S^{*}, X^{1 *}, \ldots, X^{n *}\right)$, one inverts the first relation with respect to $U^{*}$, which is possible because $\partial S^{*} / \partial U^{*}>0$. If $S^{*}$ has degree one and weights $\left(r, \alpha_{1}, \ldots, \alpha_{n}\right)$, then $U^{*}$ has degree $r$ and weights $\left(1, \alpha_{1}, \ldots, \alpha_{n}\right)$. Analogous considerations hold when passing from the energy to the entropy representation. We show that, when it is possible to invert, at least locally, a quasi-homogeneous function with respect to one variable, a quasi-homogeneous function is obtained again, having obvious degree and weights.

In the general case, let us consider a quasi-homogeneous function $w=g\left(x^{1}, \ldots, x^{n}\right)$ of degree $r$ and weights $\left(a_{1}, \ldots, a_{n}\right)$; the partial derivatives

$$
p_{i} \equiv \frac{\partial w}{\partial x^{i}}
$$

are quasi-homogeneous functions of degree $r-a_{i}$ for $i=1, \ldots, n$. Let us assume e.g. that $p_{1} \neq 0$. Then, at least locally, one can invert $w$ with respect to $x^{1}$ and find $x^{1}=h\left(w, x^{2}, \ldots, x^{n}\right)$ such that $g\left(h\left(w, x^{2}, \ldots, x^{n}\right)\right)=w$. The inverse function $h$ (where it exists and it is sufficiently smooth) is easily shown to be a quasi-homogeneous function of degree $a_{1}$ and weights $\left(r, a_{2}, \ldots, a_{n}\right)$. In fact, one has

$$
g\left(\lambda^{a_{1}} x^{1}, \lambda^{a_{2}} x^{2}, \ldots, \lambda^{a_{n}} x^{n}\right)=g\left(\lambda^{a_{1}} h\left(w, x^{2}, \ldots, x^{n}\right), \lambda^{a_{2}} x^{2}, \ldots, \lambda^{a_{n}} x^{n}\right)=\lambda^{r} g\left(x^{1}, \ldots, x^{n}\right) .
$$

Moreover, one has

$$
g\left(h\left(\lambda^{r} w, \lambda^{a_{2}} x^{2}, \ldots, \lambda^{a_{n}} x^{n}\right), \lambda^{a_{2}} x^{2}, \ldots, \lambda^{a_{n}} x^{n}\right)=\lambda^{r} w=\lambda^{r} g\left(x^{1}, \ldots, x^{n}\right),
$$

thus it has to hold

$$
h\left(\lambda^{r} w, \lambda^{a_{2}} x^{2}, \ldots, \lambda^{a_{n}} x^{n}\right)=\lambda^{a_{1}} h\left(w, x^{2}, \ldots, x^{n}\right)
$$

Cf. Ref. [35], where an analogous property is shown in passing from the entropy representation to the energy representation in standard (homogeneous) thermodynamics. See also [48].

As a further example, let us consider Kerr-Newman black holes. By inverting (where possible) the relation $T=T(M, Q, J)$ with respect to $M$, one obtains (at least formally) $M=M(T, Q, J)$. We know that $M$ and $Q$ have degree $1 / 2, J$ has degree one and $T$ has degree $-1 / 2$. In the new variables, $M=M(T, Q, J)$ is a quasi-homogeneous function of degree $1 / 2$ and weights $(-1 / 2,1 / 2,1)$. The Euler operator corresponding to the independent variables $(T, Q, J)$ is

$$
\bar{D}=-\frac{1}{2} T \frac{\partial}{\partial T}+\frac{1}{2} Q \frac{\partial}{\partial Q}+J \frac{\partial}{\partial J}
$$

and it holds

$$
\bar{D} M=\frac{1}{2} M
$$

\section{FURTHER INSIGHTS FROM BLACK HOLES}

A feature of quasi-homogeneous thermodynamics, shared also with homogeneous thermodynamics, is that in the expression of the metrical entropy and of the absolute temperature a undetermined multiplicative constant appears (cf. also [1]). Such a constant is phenomenologically fixed once the absolute scale of temperature is chosen. From the point of view of the actual analytic expression of this constant, it should be furnished by a statistical mechanical calculation. The black hole case can be again useful. By dimensional analysis, if we make the hypothesis that the entropy of a black hole can depend only on $\hbar, c, G, k_{b}$, we find that

$$
S \propto \frac{k_{b} c^{3}}{\hbar G} A
$$


this is the same procedure as in the original proposal of Bekenstein [49]. For the temperature one obtains

$$
T \propto \frac{\hbar c}{k_{b}}\left(\frac{\partial A}{\partial\left(G M / c^{2}\right)}\right)^{-1}
$$

A dimensionless constant has still to be determined.

If one considers standard thermodynamics cases, this hypothesis which allows to recover the dimensions of the entropy by involving only the above constants works for the photon gas but it does not work in other cases; e.g. in the case of a Fermi gas of electrons, the electron mass represents a further scale, related to a microscopic analysis of the system, to be taken into account. In our case, the confirmation of the above hypothesis comes from the Hawking effect, which gives us the behavior of the black hole temperature, to be compared with (115). Then, because of the absence of a particle mass scale and, e.g. in the Schwarzschild and in the Kerr cases being involved only vacuum solutions of the Einstein equations, one could be tempted to think about some sort of "graviton gas", but, actually, this is far from being evident. See also Ref. [14].

Black holes represent an example of the the procedure of reducing/enlarging consistently the thermodynamics space discussed in sect. III. In fact, the construction of the fundamental equation sketched in sect. IV is allowed also in the case of a black hole with $J=0$ and of a black hole with $Q=0$. Two parameters appear, $M, Q$ and $M, J$ respectively. The integrability condition is then trivially satisfied, but a well-defined potential $S$ is obtained everywhere. Moreover, black holes of the Kerr-Newman family are a consistent extension of the black holes of the Reissner-Nordström family (by adding $J$ ) or of the Kerr family (by adding $Q$ ). To this family belong also a thermodynamically degenerate case: the Schwarzschild case $J=0=Q$, which is described only by one variable $M$. Notice also that the Kerr-Newman family can be obtained by putting $\Lambda=0$ in the KN-AdS family.

In the case of black hole thermodynamics, it is tempting to conjecture that the quasi-homogeneous behavior of the thermodynamic potentials could be related to an explanation, to some extent, analogous to the one for the quasihomogeneous behavior of the thermodynamic potentials in standard thermodynamics near the critical point [50,51]: As in standard thermodynamics the (leading order) quasi-homogeneous behavior can be related to the conformal invariance of the underlying quantum field theory near criticality [51], one could conjecture that the quasi-homogeneous behavior of black hole thermodynamics could be related to some sort of conformal invariance of the quantum theory underlying General Relativity (superstring theory?). This field deserves further investigation. We however point out that it does not seem to be necessary that quasi-homogeneous thermodynamics should be related to some sort of conformal invariance in every case. For black holes, as well as for the general case, one should determine the reason of a quasi-homogeneous behavior by means of statistical mechanical calculations. In the following section, a further discussion is found.

\section{HEURISTICS: QUASI-HOMOGENEOUS THERMODYNAMICS AND THERMODYNAMIC LIMIT}

In this section we suggest that the thermodynamic limit, under suitable hypotheses, can lead only to quasihomogeneous thermodynamics. The thermodynamic limit should not be intended in a literal sense, of course, but as a tool which allows to determine the leading terms in the statistical mechanical functionals as large systems occur. Cf. e.g. Ref. [52], chap. 4, and also Ref. [53]. Thermodynamics of macro-systems emerges as an asymptotic law which is extrapolated from the asymptotic behavior of statistical mechanics. To some extent, the thermodynamic limit is also a tool by means of which the vague notion of "macroscopic system" is meant to be implemented, and a proper thermodynamic behavior recovered. Such a behavior depends on the interactions and on the nature of the system involved (e.g., fermionic or bosonic matter), together with a dependence on the initial conditions [from a quantum-mechanical point of view, a sort of "preparation" of the system, even if it is of "astrophysical size"]. As far as the gravitational interaction can be neglected, the standard thermodynamic behavior emerges, with the usual asymptotic laws which justify the extensivity of standard thermodynamics. At the same time, extensivity cannot be considered as an absolute and unmodifiable property of thermodynamics, because of the purely attractive nature of gravity. One can also expect a different asymptotic behavior for different systems at different scales. See also the discussion in the following subsections.

The basic set (B) of assumptions is the following:

Assumptions (B):

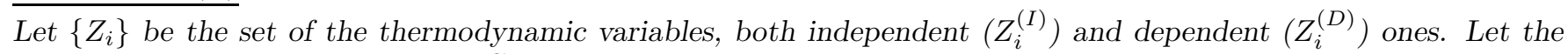
independent variables describe the Gibbs thermodynamic space.

Let $\lambda$ be the parameter such that, by taking $\lambda \rightarrow \infty$, one recovers the thermodynamic limit. 
Assume that the thermodynamic limit properly describes the bulk properties of the system under consideration. Assume that the thermodynamic limit of the statistical mechanical quantities, like e.g. the entropy or the free energy, exists under the hypothesis that, in the limit $\lambda \rightarrow \infty$, the independent thermodynamic variables $Z_{i}^{(I)}(\lambda)$ are rescaled as

$$
Z_{i}^{(I)}=g_{i}^{(I)}(\lambda) Z_{i}^{(I) 0}
$$

where $g_{i}^{(I)}(\lambda)$ is a positive function which is either an invertible function of $\lambda$ or it is one (or a constant), and $Z_{i}^{(I)} 0$ is the value of the $i$-esime variable $Z_{i}^{(I)}$ at an arbitrary reference state (cf. Ref. [53] for the extensive case).

We now state the following result (R-group).

Result (R-group):

Let us assume the set of assumptions (B) and that for the rescalings of the independent variables the group property holds:

$$
g_{i}^{(I)}(\lambda \mu)=g_{i}^{(I)}(\lambda) g_{i}^{(I)}(\mu)
$$

This implies that for each independent variable there exists a real number $\alpha_{i}^{(I)}$ such that $g_{i}^{(I)}(\lambda)=\lambda^{\alpha_{i}^{(I)}}$.

Let us assume that the dependent thermodynamic variables

$$
Z_{i}^{(D)}=Z_{i}^{(D)}\left(Z_{1}^{(I)}, \ldots, Z_{n}^{(I)}\right)
$$

are obtained as leading terms in the asymptotic expansion of the corresponding statistical mechanical functionals $\mathcal{Z}_{i}^{(D)}$ in the following sense: for each $i$ there exists a positive continuous function $\rho_{i}(\lambda)$ such that

$$
\lim _{\lambda \rightarrow \infty} \frac{1}{\rho_{i}(\lambda)} \mathcal{Z}_{i}^{(D)}\left(g_{1}^{(I)}(\lambda) Z_{1}^{(I)}, \ldots, g_{n}^{(I)}(\lambda) Z_{n}^{(I)}\right)=Z_{i}^{(D)}\left(Z_{1}^{(I)}, \ldots, Z_{n}^{(I)}\right),
$$

and that the domain of the functionals $\mathcal{Z}_{i}^{(D)}$ is invariant under the above rescalings.

Then, the dependent thermodynamic variables are quasi-homogeneous, i.e. they satisfy

$$
Z_{i}^{(D)}\left(g_{1}^{(I)}(\lambda) Z_{1}^{(I)}, \ldots, g_{n}^{(I)}(\lambda) Z_{n}^{(I)}\right)=g_{i}^{(D)}(\lambda) Z_{i}^{(D)}\left(Z_{1}^{(I)}, \ldots, Z_{n}^{(I)}\right)
$$

where $g_{i}^{(D)}(\lambda)=\lambda^{\alpha_{i}^{(D)}}$ and $\alpha_{i}^{(D)} \in \mathbb{R}$.

Moreover, for all $i$, the functions $\rho_{i}(\lambda)$ are regularly varying functions [54]:

for all $\mu \in \mathbb{R}_{+}$

$$
\lim _{\lambda \rightarrow \infty} \frac{\rho_{i}(\lambda \mu)}{\rho_{i}(\lambda)}=\mu^{\alpha_{i}^{(D)}}
$$

$\left(\alpha_{i}^{(D)}\right.$ is also called degree of $\left.\rho_{i}\right)$.

In order to be more explicit, let us consider (R-group) for a specific case. Let us e.g. assume that the thermodynamic limit for the statistical mechanical functional $\mathcal{S}$ representing the entropy exists in the sense that

$$
\lim _{\lambda \rightarrow \infty} \frac{1}{\rho_{S}(\lambda)} \mathcal{S}\left(\lambda^{\alpha_{U}} U, \lambda^{\alpha_{V}} V, \lambda^{\alpha_{N}} N\right)=S(U, V, N),
$$

where the function $\rho_{S}$ is positive and $\alpha_{U}, \alpha_{V}, \alpha_{N}$ are real numbers. Then for each real $\mu>0$ the function $\rho_{S}$ satisfies

$$
\lim _{\lambda \rightarrow \infty} \frac{\rho_{S}(\lambda \mu)}{\rho_{S}(\lambda)}=\mu^{\gamma}
$$

for some $\gamma \in \mathbb{R}$. Moreover, $S$ is quasi-homogeneous of degree $\gamma$. In fact, from (122) it follows

$$
\lim _{\lambda \rightarrow \infty} \frac{1}{\rho_{S}(\lambda \mu)} \mathcal{S}\left((\lambda \mu)^{\alpha_{U}} U,(\lambda \mu)^{\alpha_{V}} V,(\lambda \mu)^{\alpha_{N}} N\right)=S(U, V, N) .
$$


Moreover, it holds

$$
\lim _{\lambda \rightarrow \infty} \frac{1}{\rho_{S}(\lambda)} \mathcal{S}\left(\lambda^{\alpha_{U}}\left(\mu^{\alpha_{U}} U\right), \lambda^{\alpha_{V}}\left(\mu^{\alpha_{V}} V\right), \lambda^{\alpha_{N}}\left(\mu^{\alpha_{N}} N\right)\right)=S\left(\mu^{\alpha_{U}} U, \mu^{\alpha_{V}} V, \mu^{\alpha_{N}} N\right) .
$$

Then

$$
\begin{aligned}
& \lim _{\lambda \rightarrow \infty} \frac{\rho_{S}(\lambda)}{\rho_{S}(\lambda \mu)} \frac{\mathcal{S}\left((\lambda \mu)^{\alpha_{U}} U,(\lambda \mu)^{\alpha_{V}} V,(\lambda \mu)^{\alpha_{N}} N\right)}{\mathcal{S}\left(\lambda^{\alpha_{U}}\left(\mu^{\alpha_{U}} U\right), \lambda^{\alpha_{V}}\left(\mu^{\alpha_{V}} V\right), \lambda^{\alpha_{N}}\left(\mu^{\alpha_{N}} N\right)\right)}= \\
& \frac{S(U, V, N)}{S\left(\mu^{\alpha_{U}} U, \mu^{\alpha_{V}} V, \mu^{\alpha_{N}} N\right)}=\lim _{\lambda \rightarrow \infty} \frac{\rho_{S}(\lambda)}{\rho_{S}(\lambda \mu)},
\end{aligned}
$$

which is possible only if the above conditions are implemented. A general proof is found in Appendix E. Cf. [55], where a rigorous approach for asymptotics of tempered distributions is developed.

The case where one or more variables are "intensive", i.e. are not rescaled, presents no difficulty. The above reasoning about the leading order in the asymptotic law identified in the thermodynamic limit is corroborated by the standard thermodynamic limit, which allows to find a leading-order homogeneous thermodynamics. We define quasihomogeneous thermodynamic limit the thermodynamic limit satisfying the group property.

Moreover, we notice that, if e.g. for the thermodynamic entropy $S(U, V, N)$ it holds

$$
S\left(g_{U}(\lambda) U_{0}, g_{V}(\lambda) V_{0}, g_{N}(\lambda) N_{0}\right)=g_{S}(\lambda) S_{0}=g_{S}(\lambda) S\left(U_{0}, V_{0}, N_{0}\right),
$$

where the functions $g_{U}, g_{V}, g_{N}, g_{S}$ are positive and the group property holds, then $S$ has to be quasi-homogeneous and the $g_{i}$ are power-like functions. The key point is that a behavior like the one in (127) under the group property is necessarily quasi-homogeneous; as it is shown in Ref. [9], "powers need no generalization" [see Appendix A in Ref. [9] and Appendix C in Ref. [10]]. A different proof is sketched in Appendix D for the sake of completeness. The aforementioned theorem implies that actually one has

$$
S\left(g(\lambda)^{\alpha_{U}} U, g(\lambda)^{\alpha_{V}} V, g(\lambda)^{\alpha_{N}} N\right)=g(\lambda)^{\alpha_{S}} S(U, V, N)
$$

with the same scaling function $g(\lambda)$. By defining

$$
\bar{\lambda} \equiv g(\lambda)
$$

one finds $S\left(\bar{\lambda}^{\alpha_{U}} U, \bar{\lambda}^{\alpha_{V}} V, \bar{\lambda}^{\alpha_{N}} N\right)=\bar{\lambda}^{\alpha_{S}} S(U, V, N)$, i.e. the standard definition of quasi-homogeneous behavior for a function. A generalization of the quasi-homogeneous behavior of a function under rescaling with the group property is not allowed.

We now relax the requirement for the group property, one can still make a conjecture (C-reg) which would allows a quasi-homogeneous behavior under rather general conditions.

Conjecture (C-reg):

Let us assume the set $(B)$ and that $g, g_{i}$ (for $i=1, \ldots, n$ ) are positive and continuous functions which are regularly varying functions of real degree $\gamma, \alpha_{1}, \ldots, \alpha_{n}$ respectively.

Assume that the statistical mechanical functional $\mathcal{Z}$ satisfies

$$
\lim _{\lambda \rightarrow \infty} \frac{1}{g(\lambda)} \mathcal{Z}\left(g_{1}(\lambda) Z^{1}, \ldots, g_{n}(\lambda) Z^{n}\right)=Z\left(Z^{1}, \ldots, Z^{n}\right),
$$

where $Z^{i}$ are the independent thermodynamic variables (we omit the suffix $(I)$ ).

We conjecture that, under suitable hypotheses, the asymptotic $Z\left(Z^{1}, \ldots, Z^{n}\right)$ is quasi-homogeneous of degree $\gamma$ and weights $\left(\alpha_{1}, \ldots, \alpha_{n}\right)$.

For each positive real $\mu$ one should find

$$
\begin{aligned}
& \lim _{\lambda \rightarrow \infty} \frac{1}{g(\lambda \mu)} \mathcal{Z}\left(g_{1}(\lambda \mu) Z^{1}, \ldots, g_{n}(\lambda \mu) Z^{n}\right)=Z\left(Z^{1}, \ldots, Z^{n}\right) \\
& "=" \lim _{\lambda \rightarrow \infty} \frac{1}{g(\lambda) \mu^{\gamma}} \mathcal{Z}\left(g_{1}(\lambda) \mu^{\alpha_{1}} Z^{1}, \ldots, g_{n}(\lambda) \mu^{\alpha_{n}} Z^{n}\right) \\
& =\frac{1}{\mu^{\gamma}} Z\left(\mu^{\alpha_{1}} Z^{1}, \ldots, \mu^{\alpha_{n}} Z^{n}\right)
\end{aligned}
$$


where "=" indicates that further hypotheses on the statistical mechanical functional should be satisfied. Notice that the case $g_{i}(\lambda)=\lambda^{\alpha_{i}}$ is a sub-case of the preceding one and requires only that the limit (130) exists and that the state $\left(\mu^{\alpha_{1}} Z^{1}, \ldots, \mu^{\alpha_{n}} Z^{n}\right)$ belongs to the domain of the statistical mechanical functional $\mathcal{Z}$.

From a physical point of view, it can be noticed that, when passing from the statistical mechanical functional $\mathcal{S}$ representing the entropy to its asymptotic $S_{s m}$ under dilatations, as in the standard thermodynamic limit, it would be not strictly necessary, a priori, to impose that $g(\lambda)=\lambda$ in order to obtain an homogeneous $S_{s m}$ [herein we indicate by $S_{s m}$ the asymptotic which is indicated by $S$ in (122). $S_{s m}$ corresponds to the statistical mechanical entropy one takes into account in sect. III C 2]. A generic regularly varying function of degree one could also be allowed (e.g., $g(\lambda)=\lambda \log (\lambda)$ could be allowed). The requirement

$$
\lim _{\lambda \rightarrow \infty} \frac{1}{\lambda} \mathcal{S}(\lambda x)=S_{s m}(x)
$$

amounts to the requirement that in some sense also $\mathcal{S}$ is extensive.

We have found that, under rather general hypotheses, the only possible outcome of the thermodynamic limit is a form of quasi-homogeneous thermodynamics. The thermodynamic limit, at the same time, is not expected to describe all possible systems; e.g., it cannot be applied to a thin (molecular thickness) metallic film [56]. Nevertheless, it can be safely applied to a huge class of macroscopic systems, and, in the non-relativistic case, it allows to conclude that the Thomas-Fermi approximation becomes exact in the limit.

In partially concluding these heuristic considerations, we point out that a relation between a so-called pseudo-extensive thermodynamics and the thermodynamic limit has been postulated in Ref. [57] and an analysis of Newtonian selfgravitating systems is made in Ref. [58]. We limit ourselves to refer the reader to the aforementioned papers.

\section{A. non-conventional thermodynamic limit}

A non-conventional thermodynamic limit has already been proposed. A very interesting discussion of the thermodynamic limit in physical systems is found in Ref. [59]. Therein, physical systems are divided into two classes each of which can be associated with its own thermodynamic limit; one can distinguish between the standard thermodynamic limit (STL) and the inhomogeneous mean field thermodynamic limit (IMFTL) which occurs e.g. in self-gravitating systems as the one of HNT model. The latter kind of thermodynamic limit can be related with the presence of long-range forces [59]. In order to identify the correct limit, the key notion of characteristic bulk length scale is introduced, which corresponds to a typical length scale $\lambda_{t y p}$ resulting from the interaction of many particles in the system. Such length scale $\lambda_{t y p}$ plays the role of characteristic invariant in the thermodynamic limit. In the case of a classical self-gravitating isothermal gas, the Jeans length $\lambda_{\text {Jeans }}$ is such a typical length. Moreover, the requirement that mean thermodynamic quantities exist almost everywhere in the thermodynamic limit is a tool for selecting the appropriate thermodynamic limit at constant $\lambda_{t y p}$ [59]. A complementarity between STL and IMFTL can also be allowed for the same system, in the sense that STL and IMFTL can describe complementary asymptotic properties for the same large but finite system (the case of classical Coulomb systems is discussed). We don't discuss further on this approach herein, we limit ourselves to point out that one obtains the same limit, in the case of classical self-gravitating matter, if one keeps $\lambda_{\text {Jeans }}$ fixed or $\xi$ fixed (cf. sect. V). Notice that $\xi$ is an intensive function with respect to the Euler operator relative to the HNT model. At an heuristic level one expects that in general there exists a physically meaningful function which is left invariant under a quasi-homogeneous thermodynamic limit and which is intensive under the corresponding Euler operator.

A non-conventional discussion of the thermodynamic limit is found also in sections 1.2 and 4.2 of Ref. [27]. Both for stable and for unstable interactions are discussed, and the criterion for selecting the limit is a comparison between the kinetic and the potential energies involved in the system. According to [28] [p. 1-3], a non-conventional thermodynamic limit has to be associated with the peculiar scaling behavior for "non-stable interactions"; the notion of "ThomasFermi thermodynamic limit" is also introduced for non-extensive systems. Note that a more general thermodynamic limit can also be allowed, where also the coupling constants are allowed to vary in the limit. Cf. [25,28,59].

\section{B. absolute weights again}

It is evident that one has a reference quantity with respect to which all the others are "weighted" in the thermodynamic limit. Then an overall undetermined scale factor $q$ is expected to appear, unless an absolute weight is known. 
In the standard homogeneous case, the problem of assigning absolute weights does not arise, they are all equal to one for extensive variables and zero for intensive ones, the thermodynamic limit is performed with the aim of studying bulk properties of the system, and the ratios $U / N$ and $V / N$ are kept constant. Roughly, one fixes the energy per particle and the volume per particle (or, equivalently, the density $N / V$ and the energy density $U / V$ ) and then performs the limit with the aim of neglecting any finite-size effect. The same is true also for a massless particle gas, like the photon gas, where the limit $U, V \rightarrow \infty, U / V=$ const. is performed.

If a strict quasi-homogeneous picture is required, then the above problem is instead present. When $N$ is available, as it is when non-zero rest-mass particles are considered, it is to some extent natural to assume that its weight is one. Such a special role of $N$ can be justified. In the model of Hertel, Narnhofer and Thirring, the existence of the Boltzmann's entropy per particle in the thermodynamic $\operatorname{limit} \lim _{N \rightarrow \infty} \log (\Omega) / N$ is ensured, in agreement with Boltzmann's postulate, which states that the entropy per particle $\log (\Omega) / N$ exists for an equilibrium system. As a consequence of the coincidence of $\lim _{N \rightarrow \infty} \log (\Omega) / N$ with the thermodynamic entropy density (per particle), one can justify a posteriori the assumption that $N$ has weight one, in fact $\log (\Omega)$ in the thermodynamic limit coincides with the thermodynamic entropy which has degree one. The other weights $\alpha_{i}$ appropriate to the physical condition one is considering for the system are recovered in the asymptotic limit. Then, no ambiguity for an overall multiplicative constant in the definition of the weight like the one discussed in sect. III C appears. Note also that the Boltzmann's entropy, being the logarithm of the number of micro-states compatible with a given macro-state, by involving the (vague) concept of macro-state and thus of macroscopic system can legitimately related to the thermodynamic limit (again in the sense of asymptotic law). [About Boltzmann's entropy and its coincidence with the Gibbs entropy (which, in the quantum case, corresponds to the von Neumann entropy) see e.g. [60].] A more puzzling case is represented by a self-gravitating massless gas, but weights can be still fixed. See sect. VI.

In general, if $\lambda \rightarrow \infty$ corresponds to the thermodynamic limit, one could also require the existence of the Boltzmann entropy density functional as $\lambda \rightarrow \infty$, to be defined as

$$
s_{\text {boltz }} \equiv \lim _{\lambda \rightarrow \infty} \frac{1}{\lambda^{\alpha_{S}}} \log (\Omega),
$$

where $\alpha_{S}$ is a constant which is fixed by the requirement of finiteness of the above limit. $\alpha_{S}$ has to be introduced because the scale $\lambda$ can also be such that $\lambda / N$ is not asymptotically a positive constant, i.e., $\lambda \nsim N$ in the sense of the asymptotic behavior. This more general choice for the scale is also necessary if $N$ is not a good thermodynamic variable (as in the massless gas case). We know that it is consistent to fix the weights by choosing the Boltzmann entropy to be of degree one.

If another variable, e.g. the internal energy, would be used as reference variable, the relative weights could be still recovered in line of principle, but the aforementioned ambiguity would emerge in absence of a criterion for finding the absolute weight at least of one (independent or dependent) variable.

One could also choose to work in the energy representation, and decide to fix the weight of the internal energy to be one. Then the entropy in general would not be a degree one quasi-homogeneous function; in the HNT model, one would obtain $\operatorname{deg}(V)=-3 / 7 ; \operatorname{deg}(N)=3 / 7 ; \operatorname{deg}(S)=3 / 7$, against the weight $7 / 3$ of $U$ resulting by assuming that $N$ has weight one. This procedure, although legitimate because of the overall ambiguity, is less satisfactory, because the thermodynamic construction, in the case the entropy is a quasi-homogeneous function and the weights present the overall factor ambiguity, allows naturally the thermodynamic entropy to be of degree one. The only exception which, in line of principle could be admitted, is given by the case where the weights in the Gibbs space are all fixed and the statistical-mechanical entropy is not of degree one, but it is apparently not allowed (unless some reason for fixing the absolute weight of a variable appears).

\section{C. thermodynamics, weights and gravity}

As far as gravity is negligible, one finds the standard extensivity property for the fundamental equation in the entropy [energy] representation; when the scale of the system is such that is no more possible to neglect self-gravity effects, then the weights of the thermodynamic variables change according to the nature of the system and to the scale itself. The quasi-homogeneous character of thermodynamics with fixed weights $\left(\alpha_{1}, \ldots, \alpha_{n}\right)$ has to be intended as relative to the validity range of the asymptotic expansion which leads to those particular $\left(\alpha_{1}, \ldots, \alpha_{n}\right)$, whose nature is thus non absolute. [One could also be tempted to write $\alpha_{i}=\alpha_{i}(\lambda)$, in order to recall that the weights can change with the scale. Nevertheless, in light of the above theorem about quasi-homogeneity, such a dependence is not allowed. The quasi-homogeneity is extrapolated at each fixed scale, and it is made "absolute" by the thermodynamic formalism.] Also an estimate of the error for finite $\lambda$ should be allowed [52]. One could think also to a sort of "evolving picture" 
for the formalism, in which one starts from standard homogeneous systems, characterized by stable interactions, and then, as far as gravity becomes non-negligible, a quasi-homogeneous behavior is allowed. Weights can change, even in a discontinuous way, depending on the physical conditions leading to the new stationary equilibrium state. Because of the Hawking effect, the "end of the story" does not occur even when the scale and the physical conditions are such that a black hole forms, whose "classical" weights are the ones presented in sect. IV.

It is interesting to underline again that ordinary thermodynamics implements the picture described above, as discussed in several fundamental papers on the stability of matter $[61,62,29,63]$. See also Refs. [64,43]. Strict quasi-homogeneity is expected to be effective when gravitation begins playing an essential role in the physics of the system. Moreover, the so-called fourth law of thermodynamics [13] should be generalized in such a way to include the general quasihomogeneous behavior of the thermodynamic variables [dependent as well as independent ones].

\section{CONCLUSIONS}

Quasi-homogeneous thermodynamics is proposed as the unifying picture for thermodynamics where both stationary black holes, fermionic non-relativistic matter, and self-gravitating electromagnetic radiation are found.

We have chosen to introduce quasi-homogeneity in thermodynamics by generalizing the formalism developed for standard homogeneous thermodynamics [1]. The basic requirement is that the Euler vector field which generates quasi-homogeneous dilatations in the Gibbs' thermodynamic space is a non-trivial symmetry for the integrable Pfaffian form $\delta Q_{r e v}$. This means that the symmetry maps leaves of the thermodynamic foliation onto other leaves. The leaves of the foliation are naturally associated with a quasi-homogeneous function $S^{*}$ of degree one, which is suggested to be the (unique apart from a multiplicative constant) metrical entropy corresponding to the fundamental equation in the entropy representation. This is true also in the case one knows the weights of the thermodynamics variables apart from an overall unknown factor. Notice that quasi-homogeneity could be introduced also at the level of the fundamental equation in the entropy representation or in the energy one, but, in the case where only the relative weights of the independent thermodynamic variables are known, it should be considered equivalent the choice of a degree one internal energy with respect to the choice of a degree one entropy (cf. also [9]), whereas the aforementioned approach privileges a degree one entropy.

The consequences of the lack of homogeneity are recalled, and superadditivity of the metrical entropy is a privileged property ensuring the second law even in absence of concavity for $S^{*}$.

The generalized Gibbs-Duhem identities are also derived, and the energy representation and the behavior under Legendre transforms is analyzed.

An heuristic argument which can relate quasi-homogeneous thermodynamics to the thermodynamic limit in statistical mechanics (even if in a non-conventional framework) has been introduced. According to this argument, under the group property only a form of quasi-homogeneous thermodynamics can be recovered in the thermodynamic limit. Moreover, a mean field thermodynamic limit appears to be appropriate; the suggestion from the existing models is that one has to search for asymptotic scaling properties of the equations allowing to find out the mean field thermodynamics for the system, in fact the scaling properties of a finite temperature Thomas-Fermi equation appears to be involved in the quasi-homogeneity of the HNT model, whereas the scaling properties of the TOV equations are related with the quasi-homogeneity of self-gravitating radiation.

Gravity appears to play a fundamental role in allowing a generalization of the standard scheme for thermodynamics. The purely attractive nature of gravity, as well-known, is the reason for the failure of the extensivity property in thermodynamics of self-gravitating systems, because of the absence of saturation which leads to an implosive instability, and matter systems eventually can implode into a black hole if the mass is sufficiently large. Moreover, the thermodynamic ensembles become inequivalent [28], and the lack of concavity leads to phase transitions in matter systems in the canonical ensemble $[27,28]$. Negative heat capacities arise in the micro-canonical ensemble. Also black holes present this feature.

Black hole thermodynamics requires some special comments. From the point of view of thermodynamic formalism, black hole thermodynamics can be realized to be a rather straightforward generalization of the formalism developed for standard thermodynamics in [1]; moreover, its quasi-homogeneous behavior can no more be considered as exceptional, because self-gravitating systems exist where quasi-homogeneous scaling laws are satisfied. Nevertheless, from other points of view black hole thermodynamics is still to be considered special, also because it is still unclear which statistical mechanics should lie beyond it.

We limit ourselves herein to this proposal for a generalization of the thermodynamic formalism, without pursuing it further on [apart from a note on the third law in Appendix B]. For future investigations, it would be interesting to 
develop a statistical mechanical formalism where General Relativity is self-consistently included.

It would be also interesting to explore, if other long range forces are allowed to be included in the framework of quasi-homogeneous thermodynamics. Classical two-component Coulomb matter seems to belong to this framework [59], further investigations are required in order to see if this is true also for quantum Coulomb matter. 


\section{APPENDIX A: INTEGRAL OF $\omega / \mu$ AND POTENTIALS ASSOCIATED WITH QUASI-HOMOGENEOUS EXACT PFAFFIAN FORMS}

We first present some results in the general case of a generic symmetry $X$ for a Pfaffian form $\omega$. Then we translate the results for the case of the quasi-homogeneity symmetry. We recall that a vector field $X$ is defined to be a symmetry for a Pfaffian form $\omega$ if

$$
\left(L_{X} \omega\right) \wedge \omega=0 .
$$

This means that there exists a function $h$ such that

$$
L_{X} \omega=h \omega .
$$

See $[5,6]$.

We need the following two lemmas:

$\underline{\text { lemma } 1}$

Let $\bar{\omega}$ be an exact Pfaffian form;

let $X$ be a symmetry such that

$$
L_{X} \bar{\omega}=q \bar{\omega},
$$

where $q \neq 0$ is a constant. Then, one finds that $\bar{\omega}=d \phi$ is implemented by

$$
\phi \equiv \frac{1}{q} i_{X} \bar{\omega}
$$

proof: The proof is elementary. One has trivially

$$
d \phi=\frac{1}{q} d\left(i_{X} \bar{\omega}\right)=-\frac{1}{q} i_{X}(d \bar{\omega})+\frac{1}{q} L_{X} \bar{\omega} .
$$

From $d \bar{\omega}=0$ because $\bar{\omega}$ is closed and from the symmetry of $\bar{\omega}$, which implies $L_{X} \bar{\omega}=q \bar{\omega}$ the thesis follows. Notice that $\phi$ satisfies $X \phi=q \phi$.

\section{$\underline{\text { lemma } 2}$}

Let $\omega_{(0)}$ be an exact Pfaffian form;

let $X$ be a symmetry such that $L_{X} \omega_{(0)}=0$;

let $\hat{W}$ be the associated potential: $d \hat{W} \equiv \omega_{(0)}$.

Then

$$
d \hat{W}=\frac{d F}{F}
$$

where $F$ satisfies

$$
X F=q F
$$

with $q \in \mathbb{R}$ constant. Moreover,

$$
i_{X} \omega_{(0)}=q .
$$

As a consequence, $q=0$ is allowed if and only if the symmetry is tangent (i.e. trivial).

proof: We have

$$
0=L_{X} \omega_{(0)}=L_{X} d \hat{W}=d L_{X} \hat{W}
$$


As a consequence,

$$
L_{X} \hat{W}=X \hat{W}=q
$$

where $q$ is a constant. We can define a positive definite function $F$ such that

$$
\hat{W} \equiv \log (F) .
$$

Then we get that (A10) is equivalent to the following equation for $F$ :

$$
X F=q F
$$

moreover

$$
d \hat{W}=\frac{d F}{F},
$$

and

$$
i_{X} \omega_{(0)}=i_{X} d \hat{W}=L_{X} \hat{W}=\frac{1}{F} X F=q
$$

If the symmetry is tangent, then $i_{X} \omega_{(0)} \equiv 0$. Then $q=0$ necessarily. If $q=0$, then $F$ and $\hat{W}$ satisfy $X F=0$ and $X \hat{W}=0$ respectively. As a consequence, $i_{X} \omega_{(0)}=i_{X} d \hat{W}=L_{X} \hat{W}=0$, which completes the proof.

Notice that $\hat{W}$ has to be found by quadratures, the contraction of the Pfaffian form with the vector field $X$ is not useful in order to find a potential without explicit integration.

One can in general show that the following theorem holds: 
Let $\omega$ be a integrable Pfaffian form which is defined in a connected, simply connected domain;

let $X$ be a non-trivial symmetry for $\omega$;

let $\mu=i_{X} \omega$.

Then $\mu$ is an integrating factor for $\omega$ and the foliation corresponding to the Pfaffian equation $\omega=0$ is associated with a potential $F$ which satisfies the following equation:

$$
X F=F .
$$

proof: The proof that $\omega / \mu$ is closed is trivial. See also [6] and [1]. Moreover

$$
L_{X} \frac{\omega}{\mu}=0,
$$

being $X$ a symmetry for $\omega$. In fact,

$$
\begin{aligned}
L_{X} \frac{\omega}{\mu} & =\frac{1}{\mu^{2}}\left(\left(L_{X} \omega\right) \mu-\left(L_{X} \mu\right) \omega\right)=\frac{1}{\left(i_{X} \omega\right)^{2}}\left(\left(L_{X} \omega\right)\left(i_{X} \omega\right)-\left(L_{X} i_{X} \omega\right) \omega\right) \\
& =\frac{1}{\left(i_{X} \omega\right)^{2}} i_{X}\left(\omega \wedge\left(L_{X} \omega\right)\right),
\end{aligned}
$$

where the rules $i_{X} L_{X}=L_{X} i_{X}$ and $i_{X}(\alpha \wedge \beta)=\left(i_{X} \alpha\right) \wedge \beta+(-)^{n} \alpha \wedge\left(i_{X} \beta\right)$ for a $n$-form $\alpha$ and a $m$-form $\beta$ are used. Cf. [65].

As a consequence, $\omega / \mu$ is an exact one-form such that the previous lemma applies. One has

$$
\frac{\omega}{\mu}=d \hat{W}=\frac{d F}{F}
$$

where $F$ satisfied eqn. (A7) with $q \neq 0$, because of the hypothesis of non-trivial symmetry. Then one finds

$$
d F=\frac{F}{\mu} \omega,
$$

which satisfies $L_{X} d F=q d F$ and is closed. Then, from (A4), we obtain

$$
F=\frac{1}{q} \frac{F}{\mu} i_{X} \omega
$$

which, because of the definition of $\mu$, implies $q=1$.

Notice that the integrating factor satisfies

$$
L_{X} \mu=L_{X} i_{X} \omega=i_{X} L_{X} \omega=h \mu .
$$

It is interesting to underline that this theorem allows to construct a general theory of thermodynamics if $\delta Q_{\text {rev }}$ has a non-trivial symmetry. It is easy to see that the constructions of sects. III A and III B remain unaltered, and also the constructive assumptions of sect.VIII can be trivially generalized [it is sufficient to delete "quasi-homogeneous" in a1) and a2) and to change a5) into "the metrical entropy $S$ satisfies $X S=S$ ]. The case of quasi-homogeneous symmetry is immediately obtained by requiring that each independent variable in the thermodynamic space has the same kind of symmetry as the potential $S$. More general symmetries can also be introduced. Further details will be given elsewhere [66].

\section{1. the quasi-homogeneous case}

The following results are immediate consequences of the previous lemmas and theorem in the case of quasihomogeneous symmetry.

$\underline{\text { result 1: }}$ 


$$
\omega=\sum_{i=1}^{n} \omega_{i} d x^{i}
$$

be an exact quasi-homogeneous Pfaffian form of degree $r \neq 0$ and weights $\left(\alpha_{1}, \ldots, \alpha_{n}\right)$;

let

$$
D \equiv \sum_{i=1}^{n} \alpha_{i} x^{i} \frac{\partial}{\partial x^{i}}
$$

be the Euler operator;

let the symmetry be nontrivial (or transversal), i.e.

$$
\omega(D)=i_{D} \omega=\sum_{i=1}^{n} \alpha_{i} \omega_{i} x^{i} \not \equiv 0 ;
$$

Then, one finds that $\omega=d \phi$ is implemented by

$$
\phi \equiv \frac{1}{r} \omega(D)
$$

The above result can be used even when some zero weights occur. It cannot be used if $r=0$, in which case the potential can be found only by quadratures. When one considers e.g. $\delta Q_{r e v} / f$, the potential has to be found by integrating the exact form.

$\underline{\text { result } 2:}$

Let $\omega_{(0)}$ be an exact quasi-homogeneous Pfaffian form of degree $r=0$;

let $W$ be the associated potential: $d \hat{W} \equiv \omega_{(0)}$.

Then

$$
d \hat{W}=\frac{d F}{F}
$$

where $F$ is a quasi-homogeneous function of degree $q$ with respect to the Euler vector field $D$. Moreover,

$$
i_{D} \omega_{(0)}=q .
$$

As a consequence, $q=0$ is allowed if and only if the symmetry is tangent.

Notice that $\hat{W}$ has to be found by quadratures, the contraction of the Pfaffian form with the Euler vector field is not useful in order to find a potential without explicit integration.

$\underline{\text { result 3: }}$

If $\omega_{(r)}$ is a quasi-homogeneous integrable Pfaffian form of degree $r$ defined in a connected, simply connected domain, if the symmetry is non-trivial and if $\mu$ is the corresponding integrating factor $\omega(D)$, then

$$
d \hat{W} \equiv \frac{\omega_{(r)}}{\mu}=\frac{d F}{F}
$$

where $F$ is a quasi-homogeneous function of degree one with respect to the Euler vector field $D$.

The degree $r$ of $\omega_{(r)}$ can also be zero in the case where at least one weight, say $\alpha_{1}$, is different from zero (the would-be intensive variable $\omega_{1}$ has then weight $\left.-\alpha_{1}\right)$. Herein examples are given where $0<r<\infty$. It would be interesting to see if $r \leq 0$ is physically meaningful.

Notice that the fact that the case $q=0$ is not allowed by the requirement of non-triviality for the symmetry can be verified by direct inspection, in fact from eqn. (A20) it follows 


$$
\partial_{i} F=\frac{F}{\mu} \omega^{i} \quad \forall i=1, \ldots, n
$$

thus $D F=0$ becomes

$$
\sum_{i=1}^{n} \alpha_{i} x^{i} \frac{F}{\mu} \omega^{i}=0,
$$

which is not allowed, in fact, because of $\mu=\sum_{i=1}^{n} \alpha_{i} x^{i} \omega^{i}$, it would imply $F \equiv 0$. It is interesting to note that, from a mathematical point of view, $\hat{W}$ is an almost quasi-homogeneous function of degree zero with first order deficiency index identically equal to one (cf. [3]), i.e. its behavior under quasi-homogeneous scaling is

$$
\hat{W}\left(\lambda^{\alpha_{1}} x^{1}, \ldots, \lambda^{\alpha_{n}} x^{n}\right)=\hat{W}\left(x^{1}, \ldots, x^{n}\right)+\log (\lambda) .
$$

This property of $\hat{W}$ is to be related with the non-triviality of the symmetry, which allows to find an integrating factor $\mu \not \equiv 0$.

\section{2. some more results on quasi-homogeneous integrable Pfaffian forms}

We have found that, for non-trivial symmetry,

$$
\omega_{(r)}=g_{(r-1)} d F
$$

where $g_{(r-1)}$ is quasi-homogeneous of degree $r-1$. If $G$ is a quasi-homogeneous function of degree $q$ such that

$$
\omega_{(r)}=g_{(r-q)} d G
$$

then necessarily

$$
G=\zeta F^{q}
$$

where $\zeta=$ const. In fact, one has

$$
\omega_{(r)}=g_{(r-q)} d G=g_{(r-1)} d F
$$

and

$$
g_{(r-q)}=\frac{g_{(r-1)}}{d G / d F} .
$$

Moreover, the quasi-homogeneity of $G(F)$ implies

$$
D G=q G=\frac{d G}{d F} F
$$

that is,

$$
\frac{d G}{d F}=q \frac{G}{F}
$$

whose solution is (A35). For $q=1$, one obtains that the quasi-homogeneous function $F$ of degree one implementing (A33) is unique apart from a multiplicative constant. [Notice that this result can be generalized easily to the case of a generic symmetry $X$. By referring to theorem 1 , if $G$ satisfies $X G=q G$ and $\omega=b d G$, then $G=G(F)$, where $F$ is the potential (A15), and $G=\kappa F^{q}$, where $\kappa$ is a constant].

It is evident that, if $D$ is a symmetry generator, $D_{q}=q D$ is another symmetry generator for $q \in \mathbb{R}-\{0\}$. It is also interesting that, by changing $q$, the integrating factor $f_{q}$ changes in such a way that $\omega / f_{q}=d \log \left(F_{q}\right)$, where $F_{q}=F^{1 / q}$ is quasi-homogeneous of degree one with respect to $D_{q}$ (i.e., $D_{q} F_{q}=F_{q}$ ). In fact, one has $f_{q}=q f$ and

$$
\frac{\omega}{f_{q}}=\frac{1}{q} \frac{\omega}{f}=\frac{1}{q} d \log (F)=d \log \left(F^{1 / q}\right) ;
$$

then, $D_{q} F^{1 / q}=q D F^{1 / q}=q 1 / q F^{1 / q-1} D F=F^{1 / q}$. 


\section{APPENDIX B: $S$ AS A FUNCTION OF $T$ IN QUASI-HOMOGENEOUS THERMODYNAMICS AND THE THIRD LAW IN ENTROPIC FORM}

We assume that the metrical entropy $S^{*}$ is quasi-homogeneous of degree one, and that $\alpha, \alpha_{1}, \ldots, \alpha_{n}$ are the weights of the independent variables $U^{*}, X^{1 *}, \ldots, X^{n *}$. The temperature is then a quasi-homogeneous function of degree $\alpha-1$. Let us consider $S^{*}=S^{*}\left(T^{*}, X^{1 *}, \ldots, X^{n *}\right)$. The entropic form of the third law, for standard thermodynamics, states that $S \rightarrow S_{0}$ as $T \rightarrow 0$, where $S_{0}$ is a constant which has to be zero [1]. Planck's restatement of the third law $S \rightarrow 0$ as $T \rightarrow 0$ is mandatory, if the third law holds, also in the case of quasi-homogeneous thermodynamics. In fact, if, for any fixed value of $X^{1 *}, \ldots, X^{n *}$ )

$$
\lim _{T^{*} \rightarrow 0} S^{*}\left(T^{*}, X^{1 *}, \ldots, X^{n *}\right)=S^{*}\left(0, X^{1 *}, \ldots, X^{n *}\right) \equiv S_{0}^{*}
$$

holds, then, from

$$
\lim _{T^{*} \rightarrow 0} S^{*}\left(\lambda^{\alpha-1} T^{*}, \lambda^{\alpha_{1}} X^{1 *}, \ldots, \lambda^{\alpha_{n}} X^{n *}\right)=\lambda \lim _{T^{*} \rightarrow 0} S^{*}\left(T^{*}, X^{1 *}, \ldots, X^{n *}\right)=\lambda S_{0}^{*}
$$

and

$$
\lim _{T^{*} \rightarrow 0} S^{*}\left(\lambda^{\alpha-1} T^{*}, \lambda^{\alpha_{1}} X^{1 *}, \ldots, \lambda^{\alpha_{n}} X^{n *}\right)=S^{*}\left(0, \lambda^{\alpha_{1}} X^{1 *}, \ldots, \lambda^{\alpha_{n}} X^{n *}\right)=S_{0}^{*}
$$

one finds $S_{0}^{*}=0$. In the above formulas, continuity of $S$ at $T=0$ is assumed (otherwise the third law is violated $[67])$.

We know that black hole thermodynamics violates the entropic form of the third law. This behavior is not a general feature of quasi-homogeneous thermodynamics. We construct a toy-entropy which is both quasi-homogeneous and superadditive and implements the third law. Herein, as in sect. VII, with $x$ we indicate collectively the $n$ variables appearing in $S$. Our starting point consists in realizing that, given two non-negative superadditive functions $g(x), h(x)$, the function

$$
F(x) \equiv g(x) h(x)
$$

is superadditive too. In fact,

$$
\begin{aligned}
F\left(x_{1}+x_{2}\right) & =g\left(x_{1}+x_{2}\right) h\left(x_{1}+x_{2}\right) \geq\left(g\left(x_{1}\right)+g\left(x_{2}\right)\right)\left(h\left(x_{1}\right)+h\left(x_{2}\right)\right) \\
& =g\left(x_{1}\right) h\left(x_{1}\right)+g\left(x_{2}\right) h\left(x_{2}\right)+g\left(x_{1}\right) h\left(x_{2}\right)+g\left(x_{2}\right) h\left(x_{1}\right) \\
& \geq g\left(x_{1}\right) h\left(x_{1}\right)+g\left(x_{2}\right) h\left(x_{2}\right)=F\left(x_{1}\right)+F\left(x_{2}\right) .
\end{aligned}
$$

Then, let us consider $g=2 \sqrt{U^{*} X^{*}}$ and $h=X^{* 2}$. Both are superadditive functions ( $g$ is superadditive because of its homogeneity and concavity). Then $S^{*} \equiv 2 \sqrt{U^{*} X^{*}} X^{* 2}$ is superadditive and quasi-homogeneous. For $S^{*}$ as a function of $T^{*}$, one finds

$$
S^{*}=2 T^{*} X^{* 5}
$$

which satisfies $S^{*} \rightarrow 0$ as $T^{*} \rightarrow 0$.

It is also interesting to notice that, if $S$ is a strictly superadditive function everywhere (i.e., also on the boundary $T=0)$ then the entropic version of the third law in Planck's form has to be violated; in fact, if $\left(U_{0}, X_{0}^{1}, \ldots, X_{0}^{n}\right)$ is such that $T\left(U_{0}, X_{0}^{1}, \ldots, X_{0}^{n}\right)=0$, then

$$
S\left(U+U_{0}, X^{1}+X_{0}^{1}, \ldots, X^{n}+X_{0}^{n}\right)>S\left(U_{0}, X_{0}^{1}, \ldots, X_{0}^{n}\right)+S\left(U, X_{1}, \ldots, X^{n}\right)
$$

is possible with the strict inequality only if $S\left(U_{0}, X_{0}^{1}, \ldots, X_{0}^{n}\right)>0$. In the case of a self-gravitating system belonging to quasi-homogeneous thermodynamics framework, if the energy is strictly subadditive everywhere, then the third law cannot hold.

As far as mathematical properties of the Pfaffian form $\delta Q_{r e v}$ ensuring the validity of the entropic form of the third law are concerned, it can be shown that a quasi-homogeneous $\delta Q_{\text {rev }}$ which is $C^{1}$ everywhere is a sufficient condition, in analogy with standard thermodynamics $[1,67]$. In fact, a superadditive non-negative function $S: \mathcal{D} \rightarrow \mathbb{R}_{+} \cup\{\infty\}$ cannot be divergent at a limit point $x_{0}$ of its domain [we mean that all the points $x$ in the domain satisfy $\|x\|<\infty$, where $\|\cdot\|$ stays for the Euclidean norm and that $x_{0}$ can be included in the domain by putting $\left.S\left(x_{0}\right)=\infty\right]$. This is 
evident if one translates rigidly the domain in such a way that $x_{0} \equiv 0$. Then, if $z_{0}$ is another point in the domain of $S$ one has $S\left(z_{0}\right)=S\left(z_{0}+0\right) \geq S(0)+S\left(z_{0}\right)=\infty$. Thus, if $S \not \equiv \infty$, then $S$ has to be finite. Then $S$ has to be finite in the limit as $T \rightarrow 0$. As a consequence, if $f$ is the integrating factor, one has

$$
\frac{\partial f}{\partial U^{*}}=1+S^{*} \frac{\partial T^{*}}{\partial U^{*}}
$$

and $\partial T^{*} / \partial U^{*}=1 / C$, where $C$ is the heat capacity at fixed parameters $X^{i *}$, which has to tend to zero if $S^{*}$ is finite and non-negative as $T^{*} \rightarrow 0$. Then, if $f$ is everywhere $C^{1}$ (and it is such if $\delta Q_{\text {rev }}$ is $C^{1}$ everywhere), it holds $S^{*} \rightarrow 0$ as $T^{*} \rightarrow 0$. Moreover, it is evident from (18) that $\lim _{T^{*} \rightarrow 0} S^{*}=0$ holds if and only if $\int_{\gamma} \delta Q_{\text {rev }} / f^{*} \rightarrow-\infty$ as $T^{*} \rightarrow 0$ whichever path is chosen in approaching $T^{*}=0$. Even the latter is an extension to the quasi-homogeneous case of the standard case $[1,67]$. See also [68] for further conditions.

\section{APPENDIX C: GIBBS-DUHEM EQUATION REVISITED}

Let us consider a quasi-homogeneous function $g\left(x^{1}, \ldots, x^{n}\right)$ of degree $q \neq 0$ and of weights $\left(\alpha_{1}, \ldots, \alpha_{p}, 0, \ldots, 0\right)$ where $1 \leq p \leq n$ is an integer and the last $n-p$ weights are all zero. It satisfies

$$
D g=q g,
$$

where

$$
D=\sum_{i \leq p} \alpha_{i} x^{i} \frac{\partial}{\partial x^{i}}
$$

If one defines

$$
g_{i} \equiv \frac{\partial g}{\partial x^{i}} \quad \forall i=1, \ldots, n
$$

and

$$
\omega_{g} \equiv \sum_{i} g_{i} d x^{i}=d g,
$$

one finds

$$
g=\frac{1}{q} i_{D} \omega_{g} .
$$

This formal manipulation is useful in order to derive the Gibbs-Duhem equation. In fact, one has

$$
d g=d\left(\frac{1}{q} i_{D} \omega_{g}\right)=\frac{1}{q}\left(-i_{D} d \omega_{g}+L_{D} \omega_{g}\right),
$$

where $L_{D} \omega_{g}=q \omega_{g}$ holds. Moreover, $d \omega_{g}=0$ because $d g=\omega_{g}$ by construction. The Gibbs-Duhem equation is shown to be equivalent to

$$
-\frac{1}{q} i_{D} d \omega_{g}=0
$$

One can easily see that

$$
d \omega_{g}=\sum_{i} d g_{i} \wedge d x^{i}
$$

and that

$$
i_{D} d \omega_{g}=\sum_{i}\left(i_{D} d g_{i}\right) d x^{i}-\sum_{i} d g_{i}\left(i_{D} d x^{i}\right)
$$




$$
\begin{aligned}
& =\sum_{i}\left(D g_{i}\right) d x^{i}-\sum_{i \leq p} d g_{i}\left(\alpha_{i} x^{i}\right) \\
& =\sum_{i \leq p}\left(q-\alpha_{i}\right) g_{i} d x^{i}-\sum_{i \leq p} d g_{i}\left(\alpha_{i} x^{i}\right)+\sum_{p<i \leq n} q g_{i} d x^{i} .
\end{aligned}
$$

As a consequence, $(\mathrm{C} 7)$ becomes

$$
\sum_{i \leq p} \frac{\alpha_{i}}{q}\left(x^{i}\right)^{q / \alpha_{i}} d\left(\left(x^{i}\right)^{1-q / \alpha_{i}} g_{i}\right)-\sum_{p<i \leq n} g_{i} d x^{i}=0 .
$$

If none independent variable of weight zero appears, then one finds

$$
\sum_{i} \frac{\alpha_{i}}{q}\left(x^{i}\right)^{q / \alpha_{i}} d\left(\left(x^{i}\right)^{1-q / \alpha_{i}} g_{i}\right)=0
$$

Let us consider the inverse problem, where one assigns $n$ would-be intensive variables $g_{i}$ and the quasi-homogeneous 1-form of degree $\omega_{g}=\sum_{i} g_{i} d x^{i}$ such that $L_{D} \omega_{g}=q \omega_{g}$ (one has to require that $\operatorname{deg}\left(g_{i}\right)=q-\alpha_{i}$, where the weights of the independent variables $x^{i}$ are the same as above). Then one can also define a function $g \equiv i_{D} \omega_{g} / q$. From $d g=\left(-i_{D} d \omega_{g}\right) / q+\omega_{g}$ follows that $\omega_{g}$ is closed if $i_{D} d \omega_{g}=0$, i.e., if the Gibbs-Duhem equation is satisfied.

We limit ourselves to point out that more general Gibbs-Duhem equations are obtained in the generic symmetric case [66]. The generalization of (83) is

$$
\left(i_{X} \delta Q_{\text {rev }}\right) d \log \left(\frac{1}{T}\right)-i_{X} d \delta Q_{\text {rev }}+\left(L_{X}-1\right) \delta Q_{\text {rev }}=0
$$

\section{APPENDIX D: QUASI-HOMOGENEITY (SCALING) CANNOT BE GENERALIZED}

We show that, if $f\left(x^{1}, \ldots, x^{n}\right)$ is a $C^{1}$ function on a open connected set which satisfies the following identity:

$$
f\left(g_{1}(\lambda) x^{1}, \ldots, g_{n}(\lambda) x^{n}\right)=g(\lambda) f\left(x^{1}, \ldots, x^{n}\right)
$$

and the functions $g(\lambda), g_{i}(\lambda)$ for $i=1, \ldots, n$ are positive definite and invertible, then $f\left(x^{1}, \ldots, x^{n}\right)$ is necessarily quasi-homogeneous. The above transformation is meant to be obtained by a "generalized similarity transformation" which carries $x^{1}, \ldots, x^{n}$ into $g_{1}(\lambda) x^{1}, \ldots, g_{n}(\lambda) x^{n}$. Let us consider $u \equiv g(\lambda)$ and the inverse $\lambda=g^{-1}(u)$. Define

$$
h_{i}(u) \equiv g_{i}\left(g^{-1}(u)\right) \quad \forall i=1, \ldots, n .
$$

It is also useful to define

$$
u \equiv e^{s} .
$$

Then we obtain

$$
f\left(h_{1}\left(e^{s}\right) x^{1}, \ldots, h_{n}\left(e^{s}\right) x^{n}\right)=e^{s} f\left(x^{1}, \ldots, x^{n}\right) .
$$

Note that, for $s=0$, one finds

$$
f\left(h_{1}(1) x^{1}, \ldots, h_{n}(1) x^{n}\right)=f\left(x^{1}, \ldots, x^{n}\right) .
$$

This a priori does not imply that $h_{i}(1)=1 \quad \forall i=1, \ldots, n$, but the one-parameter $s \in \mathbb{R}_{+}$"generalized similarity transformation" $\mathcal{T}_{s}:\left(x^{1}, \ldots, x^{n}\right) \rightarrow\left(h_{1}\left(e^{s}\right) x^{1}, \ldots, h_{n}\left(e^{s}\right) x^{n}\right)$ is consistently defined if $\mathcal{T}_{s=0}$ is the identity, i.e. if $h_{i}(1)=1 \quad \forall i=1, \ldots, n$.

We introduce the auxiliary function

$$
F\left(s ; x^{1}, \ldots, x^{n}\right) \equiv e^{-s} f\left(h_{1}\left(e^{s}\right) x^{1}, \ldots, h_{n}\left(e^{s}\right) x^{n}\right) .
$$

It is such that $\partial_{s} F=0$, i.e. 


$$
F=\sum_{i=1}^{n} e^{s} h_{i}^{\prime}\left(e^{s}\right) x^{i}\left(\frac{\partial F}{\partial x^{i}}\right)\left(h_{1}\left(e^{s}\right) x^{1}, \ldots, h_{n}\left(e^{s}\right) x^{n}\right)
$$

where $h_{i}^{\prime}(u) \equiv d h_{i} / d u$. For $s=0$ one obtains

$$
f\left(x^{1}, \ldots, x^{n}\right)=\sum_{i=1}^{n} h_{i}^{\prime}(1) x^{i}\left(\frac{\partial f}{\partial x^{i}}\right)\left(x^{1}, \ldots, x^{n}\right) .
$$

Thus, $f\left(x^{1}, \ldots, x^{n}\right)$ is a quasi-homogeneous function of degree one and weights $\left(h_{1}^{\prime}(1), \ldots, h_{n}^{\prime}(1)\right) \equiv\left(\alpha_{1}, \ldots, \alpha_{n}\right)$. As a consequence, one has

$$
f\left(e^{\alpha_{1} s} x^{1}, \ldots, e^{\alpha_{n} s} x^{n}\right)=e^{s} f\left(x^{1}, \ldots, x^{n}\right)
$$

i.e.

$$
h_{i}\left(e^{s}\right)=e^{\alpha_{i} s} \quad \forall i=1, \ldots, n
$$

By resorting the original dependence on $\lambda$, one finds

$$
f\left((g(\lambda))^{\alpha_{1}} x^{1}, \ldots,(g(\lambda))^{\alpha_{n}} x^{n}\right)=g(\lambda) f\left(x^{1}, \ldots, x^{n}\right),
$$

i.e.

$$
g_{i}(\lambda)=(g(\lambda))^{\alpha_{i}} \quad \forall i=1, \ldots, n .
$$

If some $g_{i}=1$ above, then one simply finds that the variable $x^{i}$ is quasi-homogeneous of degree zero, i.e., it has weight zero. If instead $g(\lambda)=1$, then $f$ is quasi-homogeneous of degree zero. The proof is straightforward. Let $\lambda_{0}$ be such that $g_{i}\left(\lambda_{0}\right)=1 \quad \forall i=1, \ldots, n$. We have that

$$
\frac{\partial f}{\partial \lambda}=0=\sum_{i=1}^{n} g_{i}^{\prime}(\lambda) x^{i}\left(\frac{\partial f}{\partial x^{i}}\right)\left(g_{1}(\lambda) x^{1}, \ldots, g_{n}(\lambda) x^{n}\right) .
$$

By setting $\lambda=\lambda_{0}$, one finds

$$
\sum_{i=1}^{n} g_{i}^{\prime}\left(\lambda_{0}\right) x^{i}\left(\frac{\partial f}{\partial x^{i}}\right)\left(x^{1}, \ldots, x^{n}\right)
$$

i.e., $f$ is quasi-homogeneous of degree zero and weights $\left(g_{1}^{\prime}\left(\lambda_{0}\right), \ldots, g_{n}^{\prime}\left(\lambda_{0}\right)\right)$.

\section{APPENDIX E: SCALING AND ASYMPTOTICS}

Let us assume that $f(x)$ is a function of $n$ variables collectively indicated with $x$. Let the domain of $f$ be invariant under quasi-homogeneous transformations. Let $\left\{\mathcal{T}_{\lambda}\right\}$ be a one-parameter quasi-homogeneous transformation. If we require that there exist a positive continuous function $\rho(\lambda)$ and a continuous function $g(x) \not \equiv 0$ such that $g$ is an asymptotic of $f$ in the following sense:

$$
\lim _{\lambda \rightarrow \infty} \frac{1}{\rho(\lambda)} f\left(\mathcal{T}_{\lambda} x\right)=g(x)
$$

where the limit is assumed to exist. Then:

a) the function $\rho(\lambda)$ is a regularly varying function, i.e. it satisfies for all $a>0$

$$
\lim _{\lambda \rightarrow \infty} \frac{\rho(\lambda a)}{\rho(\lambda)} \equiv C(a)
$$

where $C(a) C(b)=C(a b)$, i.e. $C(a)=a^{\gamma}$ for some $\gamma \in \mathbb{R} ; \gamma$ is also defined the order of the regularly varying function $\rho(\lambda)$;

b) the function satisfies the "homogeneity" relation 


$$
g\left(\mathcal{T}_{\mu} x\right)=\mu^{\gamma} g(x)
$$

i.e. $g(x)$ is quasi-homogeneous of degree $\gamma$.

proof: for each real $s>0$ it holds

$$
\lim _{\lambda \rightarrow \infty} \frac{1}{\rho(\lambda s)} f\left(\mathcal{T}_{\lambda s} x\right)=g(x)
$$

and, because of $\mathcal{T}_{\lambda s}=\mathcal{T}_{\lambda} \mathcal{T}_{s}$

$$
\lim _{\lambda \rightarrow \infty} \frac{1}{\rho(\lambda)} f\left(\mathcal{T}_{\lambda}\left(\mathcal{T}_{s} x\right)\right)=g\left(\mathcal{T}_{s} x\right)
$$

As a consequence,

$$
\lim _{\lambda \rightarrow \infty} \frac{\rho(\lambda)}{\rho(\lambda s)} \frac{f\left(\mathcal{T}_{\lambda s} x\right)}{f\left(\mathcal{T}_{\lambda}\left(\mathcal{T}_{s} x\right)\right)}=\frac{g(x)}{g\left(\mathcal{T}_{s} x\right)}=\lim _{\lambda \rightarrow \infty} \frac{\rho(\lambda)}{\rho(\lambda s)} .
$$

This is possible only if the following conditions

$$
\lim _{\lambda \rightarrow \infty} \frac{\rho(\lambda s)}{\rho(\lambda)}=C(s)
$$

and

$$
g\left(\mathcal{T}_{s} x\right)=C(s) g(x)
$$

are both satisfied. Moreover, it is easy to see that it holds

$$
C(s t)=C(s) C(t)
$$

which implies that there exists a real number $\gamma$ such that $C(s)=s^{\gamma}$. Thus, one obtains

$$
g\left(\mathcal{T}_{s} x\right)=s^{\gamma} g(x),
$$

which concludes the proof.

Cf. also Ref. [55].

[1] F.Belgiorno, Homogeneity as a Bridge between Gibbs and Carathéodory. math-ph/0210011 (2002).

[2] D.V.Anosov et al., Ordinary Differential Equations and Smooth Dynamical Systems. Springer-Verlag, Berlin (1997).

[3] Olaf von Grudzinski, Quasihomogeneous Distributions. North-Holland Mathematics Studies 165, North-Holland, Amsterdam, (1991).

[4] L.Dresner, Applications of Lie's Theory of Ordinary and Partial Differential Equations. Institute of Physics Publishing, Bristol and Philadelphia (1999).

[5] A.V.Bocharov et al., Symmetries and Conservation Laws for Differential Equations of Mathematical Physics. Translations of Mathematical Monographs 182, American Mathematical Society, Providence, Rhode Island (1999).

[6] D.Cerveau and J-F.Mattei, Formes intégrables holomorphes singulières. Astérisque 971 (1982).

[7] T.Rockafellar, Convex Analysis, Princeton University Press, Princeton (1970).

[8] P.T.Landsberg and D.Tranah, Phys. Lett 78A, 219 (1980).

[9] A.Hankey and H.E.Stanley, Phys. Rev. B6, 3515 (1972).

[10] T.S.Chang, A.Hankey and H.E.Stanley, Phys. Rev. B8, 346 (1973).

[11] V.D.Neff, Phys. Rev. B9, 3153 (1974).

[12] J.Aczél, Lectures on Functional Equations and their Applications. Academic, New York (1966).

[13] P.T.Landsberg, Jou. Stat. Phys. 35, 159 (1984).

[14] F.Belgiorno, Black Hole Thermodynamics in Carathéodory's Approach. gr-qc/0210020 (2002). 
[15] R.Wald, Quantum Field Theory in Curved Spacetime and Black Hole Thermodynamics. Chicago University Press, Chicago (1994).

[16] I.D.Novikov,V.P.Frolov, Physics of Black Holes. Kluwer Academic Publishers, Dordrecht (1989).

[17] F.Belgiorno and M.Martellini, Black Holes and the Third Law of Thermodynamics. gr-qc/0210026 (2002).

[18] P.C.W.Davies, Proc. R. Soc. Lond. A353, 499 (1977).

[19] B.Carter, in Black Holes. B.DeWitt and C.DeWitt eds. Gordon and Breach, New York (1973).

[20] G.W.Gibbons and S.W.Hawking, Phys. Rev. Lett. 15, 2752 (1977).

[21] Euclidean Quantum Gravity. G.W.Gibbons and S.W.Hawking editors, World Scientific, Singapore (1993).

[22] P.T.Landsberg and D.Tranah, Collect. Phenom. 3, 73 (1980).

[23] D.Tranah and P.T.Landsberg, Collect. Phenom. 3, 81 (1980).

[24] M.M.Caldarelli, G.Cognola and Dietmar Klemm, Class. Quant. Grav. 17, 399 (2000).

[25] P.Hertel, H.Narnhofer, W.Thirring, Commun. Math. Phys. 28, 159 (1972).

[26] P.Hertel and W.Thirring, Commun. Math. Phys. 24, 22 (1971).

[27] W. Thirring, A Course in Mathematical Physics. Vol 4. Quantum Mechanics of Large Systems. Springer-Verlag, New York (1980). Especially sect. 4.2.

[28] J.Messer, Temperature Dependent Thomas-Fermi Theory. Lecture Notes in Physics 147. Springer-Verlag, New York (1981).

[29] Jean-Marc Lévy-Leblond, Jou. Math. Phys. 10, 806 (1968).

[30] H.J.de Vega, N. Sanchez, Nucl. Phys. B625, 409 (2002).

[31] N.Bilić and R.D.Violler, Gen. Rel. Grav. 31, 1105 (1999).

[32] E.A.Power and John A.Wheeler, Rev. Mod. Phys. 29, 480 (1957).

[33] John A.Wheeler, Phys. Rev. 97, 511 (1955).

[34] R.D.Sorkin, R.M.Wald and Z.Z.Jiu, Gen. Rel. Grav. 13, 1127 (1981).

[35] L.Galgani and A.Scotti, Pure Appl. Chem. 22, 229 (1970);

L.Galgani and A.Scotti, Proceedings of the International Conference on Thermodynamics. Cardiff, P.T.Landsberg ed. Butterworths, London (1970).

[36] P.T.Landsberg, Proceedings of the International Conference on Thermodynamics. Cardiff, P.T.Landsberg ed. Butterworths, London (1970).

[37] W.Thirring, introduction to Ref. [43]. pp. 1-8.

[38] W.Thirring, in Fundamental Aspects of Quantum Theory. NATO ASI Series. Series B, Physics; 144. V.Gorini and A.Frigerio eds. Plenum, New York, (1986). pp. 343-354.

[39] D.Lynden-Bell and R.M.Lynden-Bell, Monthly Notices Roy. Astron. Soc. 181, 405 (1977).

[40] W.Thirring, Z. Phys. 235, 339 (1971).

[41] T.Padmanabhan, Phys. Rep. 188, 285 (1990).

[42] P.-H. Chavanis, C.Rosier and C.Sire, Thermodynamics of self-gravitating systems. Preprint, cond-mat/0107345 (2001).

[43] The Stability of Matter: From Atoms to Stars. Selecta of Elliott H. Lieb. Edited by W.Thirring. Springer-Verlag, Berlin, (2001).

[44] E.H. Lieb, Rev. Mod. Phys. 48, 553 (1976).

[45] E.H. Lieb and J.L.Lebowitz, Adv. Math. 9,316 (1972).

[46] H.B. Callen, Thermodynamics and an Introduction to Thermostatistics. Wiley, New York (1985).

[47] F.Belgiorno, Notes on Quasi-homogeneous Functions in Thermodynamics. physics/0210031 (2002).

[48] P.T.Landsberg, Thermodynamics and Statistical Mechanics, Dover, New York, (1990).

[49] J.D.Bekenstein, Phys. Rev. D7, 2333 (1973).

[50] D.I.Uzunov, Theory of Critical Phenomena. World Scientific, Singapore (1993).

[51] M.Henkel, Conformal Invariance and Critical Phenomena. Springer-Verlag, Berlin (1999).

[52] A.Münster, Statistical Thermodynamics, Springer Academic Press, New York, (1969).

[53] E.Still, K.Haubold, and A.Münster, Z. Naturforsch. 24 a, 201 (1969); ibid., 412 (1969).

[54] E.Seneta, Regularly Varying Functions. Lecture Notes in Mathematics 508, Springer-Verlag, Berlin, (1976).

[55] V.S.Vladimirov, B.I.Zavjalov and J.N.Drozzinov, Tauberian Theorems for Generalized Functions. Mathematics and its Applications (Soviet Series) 10, Kluwer Academic Publishers, Dordrecht, (1988).

[56] R.Balescu, Equilibrium and Nonequilibrium Statistical Mechanics. Wiley, New York (1975).

[57] L.Velasquez and F.Guzman, Extending the Equilibrium Thermodynamic Formalism to some nonextensive systems. condmat/0107439 (2001).

[58] L.Velasquez and F.Guzman, Astrophysical Systems: A model based on the self-similarity scaling postulates. condmat/0205085 (2002).

[59] M. K.-H. Kiessling, Jou. Stat. Phys. 59, 1157 (1990).

[60] S.Goldstein, Boltzmann's Approach to Statistical Mechanics. cond-mat/0105242 (2001).

[61] M.E.Fisher and D.Ruelle, J. Math. Phys. 7, 260 (1966).

[62] A.Lenard and F.J.Dyson, J. Math. Phys. 8, 423 (1967);

F.J.Dyson and A.Lenard, J. Math. Phys. 9, 698 (1968).

[63] J.L.Lebovitz and E.H.Lieb, Phys. Rev. Lett. 22, 731 (1969). 
[64] D.Ruelle, Statistical Mechanics. Rigorous Results. Benjamin, New York (1969).

[65] S.Morita, Geometry of Differential Forms. Translations of Mathematical Monographs 201, American Mathematical Society, Providence, Rhode Island (2001).

[66] F.Belgiorno, Equilibrium Thermodynamics and Symmetries of $\delta Q_{\text {rev }}$. In progress (2002).

[67] F.Belgiorno, Notes on the third law of thermodynamics.I. physics/0210037 (2002).

[68] F.Belgiorno, Notes on the third law of thermodynamics.II. To appear (2002). 